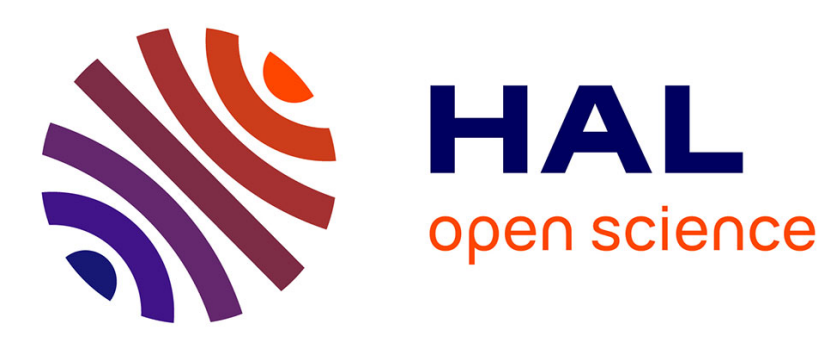

\title{
Selective focusing on small scatterers in acoustic waveguides using time reversal mirrors \\ Bruno Pinçon, Karim Ramdani
}

\section{To cite this version:}

Bruno Pinçon, Karim Ramdani. Selective focusing on small scatterers in acoustic waveguides using time reversal mirrors. Inverse Problems, 2007, 23 (1), pp.1-25. 10.1088/0266-5611/23/1/001 . hal00125005

\section{HAL Id: hal-00125005 \\ https://hal.science/hal-00125005}

Submitted on 25 Feb 2013

HAL is a multi-disciplinary open access archive for the deposit and dissemination of scientific research documents, whether they are published or not. The documents may come from teaching and research institutions in France or abroad, or from public or private research centers.
L'archive ouverte pluridisciplinaire HAL, est destinée au dépôt et à la diffusion de documents scientifiques de niveau recherche, publiés ou non, émanant des établissements d'enseignement et de recherche français ou étrangers, des laboratoires publics ou privés. 


\title{
Selective focusing on small scatterers in acoustic waveguides using time reversal mirrors
}

\author{
B Pinçon and K Ramdani \\ INRIA (Projet CORIDA) and Institut Élie Cartan de Nancy, Université Nancy \\ 1, BP 239, Vandœuvre-lès-Nancy, 54506 France. \\ E-mail: pincon@iecn.u-nancy.fr, karim.ramdani@inria.fr
}

\begin{abstract}
We investigate the acoustic selective focusing properties of time reversal in a two-dimensional acoustic waveguide. A far-field model of the problem is proposed in the time-harmonic case. In order to tackle the question of selective focusing, we derive an asymptotic model for small scatterers. We show that in the framework of this limit problem, approximate eigenvectors of the time reversal operator can be obtained when the number of propagating modes of the waveguide is large enough. This result provides in particular a mathematical justification of the selective focusing properties observed experimentally. Some numerical experiments of selective focusing are presented.
\end{abstract}




\section{Introduction}

Since the first time reversal mirrors (TRM) were conceived about fifteen years ago by the team of Mathias Fink (LOA Laboratory, France), acoustic time reversal has known a significant growth of interest. One of the main reasons of this success lies in the very wide range of applications of TRM (medical imaging, non destructive testing, underwater communication...). The physical principle underlying the phenomenon of time reversal is to use the reversibility of the wave equation in a non dissipative unknown medium to back-propagate signals towards the sources that emitted them. Today, the physical literature (cf. [11] for a detailed list of references) on this topic is quite rich. Most of the mathematical papers dealing with time reversal deal with the case of random propagation media (cf. [6] for more details). Among the very few mathematical papers dealing with time reversal in deterministic media, let us mention [5], [7] and more especially [13] and [15] for the case of time-harmonic waves considered in the present paper.

In reference [13], the first mathematical analysis of the so-called D.O.R.T. method (Decomposition of the Time Reversal Operator in french, see [12]) has been proposed. This experimental method is used to achieve selective focusing on scattering obstacles using TRM. In the case of time-harmonic waves, the way such mirrors work can be described as follows: the TRM first emits an acoustic wave in a homogeneous and non dissipative medium containing some unknown obstacles, and then measures the diffracted field. The measured field is then conjugated (reversing time amounts to a conjugation when the time dependence is of the form $\left.e^{-i \omega t}\right)$ and re-emitted. The time reversal operator $T$ is the operator obtained by iterating this procedure twice. The experimental results obtained in [12] show that for small scatterers, the number of nonzero (or significant) eigenvalues of $T$ is exactly the number of obstacles contained in the propagation medium. Furthermore, the corresponding eigenvectors generate incident waves that focus selectively on the scatterers, provided these scatterers are distant enough from each other. A rigorous mathematical justification of this phenomenon has been given in [13] when the propagation medium is the free space $\mathbb{R}^{3}$. The present work is devoted to the analysis of the case where the propagation medium is a two-dimensional waveguide $\Omega_{\infty}=\mathbb{R} \times(0, d)$. In our model, the TRM is supposed to emit a superposition of propagating modes of the waveguide, and measures the propagating part of the field diffracted by the scatterers. Our main result is that selective focusing holds true for small scatterers, provided the number $N$ of propagating modes of the waveguide is large enough. In other words, and according to relation (2.2) below, the case investigated in this paper is the one corresponding to $\varepsilon<<\lambda<<d$, where $\varepsilon$ denotes the typical size of the scatterers and $\lambda=2 \pi / k$ denotes the wavelength.

The paper is organized as follows. We start in Section 2 with the description of the mathematical model used throughout the paper for the emission and reception steps of the time reversal process. In particular, we define precisely the type of incident waves used, namely superpositions of guided modes of the waveguide. This naturally leads us to the definition of the scattering matrix $\mathbb{S}$ of the problem and the time reversal matrix $\mathbb{T}$. Section 3 is devoted to the analysis of some useful mathematical properties of these two matrices. We show in particular that the time reversal operator is selfadjoint. The selective focusing properties of the D.O.R.T. method are established in Section 4, which constitutes the core of this paper. More precisely, we first study in subsection 4.1 the asymptotic behavior of the matrices $\mathbb{S}$ and $\mathbb{T}$ for small scatterers. 
The proof of this asymptotic behavior, which is of independent interest, is given in the Appendix. Subsection 4.2 is devoted to the proof of a technical result concerning the asymptotics of a sum of exponentials. This result is used in subsection 4.3 to perform the spectral analysis of the limit model obtained in subsection 4.1 when the number $N$ of propagating modes of the waveguide is large enough. Under this assumption, we derive approximate eigenvectors of $\mathbb{T}$ and we prove that the incident field corresponding to each approximate eigenvector selectively focuses on each scatterer. Finally, some numerical experiments of selective focusing are presented in Section 5.

\section{The mathematical model}

We consider a two-dimensional acoustic waveguide $\Omega_{\infty}=\mathbb{R} \times(0, d)$ characterized by a wavenumber $k>0$. The modes of the waveguide are defined as solutions of the Helmholtz equation $\left(\Delta+k^{2}\right) U=0$ in $\Omega_{\infty}$ with homogeneous Dirichlet boundary condition. More precisely, let us introduce the eigenfunctions of the operator $-d^{2} / d \xi^{2}$ with domain $H^{2}(0, d) \cap H_{0}^{1}(0, d)$ : for all $m \in \mathbb{N}^{*}$, set

$$
\phi_{m}(\xi)=\sqrt{2 / d} \sin (m \pi \xi / d), \quad \forall \xi \in(0, d) .
$$

The eigenfunction $\phi_{m}$ is associated with the eigenvalue $(m \pi / d)^{2}$, and the family $\left(\phi_{m}\right)_{m \in \mathbb{N}^{*}}$ constitutes an orthonormal basis of $L^{2}(0, d)$. The usual scalar product on $L^{2}(0, d)$ is denoted by $(\cdot, \cdot)$ throughout the paper. We assume throughout the paper that $k$ is not a cutoff frequency, i.e. $k d / \pi \notin \mathbb{N}^{*}$. The modes are then defined as follows.

Definition 2.1. With the above notation, let $N$ be the integer defined by

$$
N=\operatorname{Card}\left\{m \in \mathbb{N}^{*} \mid m \pi / d<k\right\}=\lfloor k d / \pi\rfloor .
$$

Define the constants of propagation

$$
\beta_{m}= \begin{cases}\sqrt{k^{2}-(m \pi / d)^{2}} & \text { for } 1 \leq m \leq N, \\ i \sqrt{(m \pi / d)^{2}-k^{2}} & \text { for } m>N .\end{cases}
$$

The modes of the waveguide $\Omega_{\infty}=\mathbb{R} \times(0, d)$ are the functions defined for all $m \in \mathbb{N}^{*}$ by

$$
\Phi_{m}^{ \pm}(x)=\Phi_{m}^{ \pm}\left(x_{1}, x_{2}\right)=\exp \left( \pm i \beta_{m} x_{1}\right) \phi_{m}\left(x_{2}\right) .
$$

According to (2.3), the modes $\Phi_{m}^{ \pm}$can be decomposed into

- a finite family $\left(\Phi_{m}^{ \pm}\right)_{1 \leq m \leq N}$ of propagating modes (or guided modes) propagating towards the directions $x_{1} \rightarrow \pm \infty$, which corresponds to the case where $\beta_{m} \in \mathbb{R}$.

- a countable family $\left(\Phi_{m}^{ \pm}\right)_{m>N}$ of evanescent modes as $x_{1} \rightarrow \pm \infty$ which corresponds to the case where $\beta_{m} \in i \mathbb{R}$.

We are now in position to describe the mathematical model used for the emission and the reception steps of the time reversal procedure. The time reversal mirror is located on the boundary $\Gamma_{A}=\left\{x_{1}=0,0<x_{2}<d\right\}$ (see Figure 1). We assume that the waveguide $\Omega_{\infty}$ contains some scatterers located in the cell $(\ell, L) \times(0, d)$, where $0<\ell<L$. Let $\mathcal{O}$ be the open set occupied by the scatterers and $\Gamma$ be its boundary. 


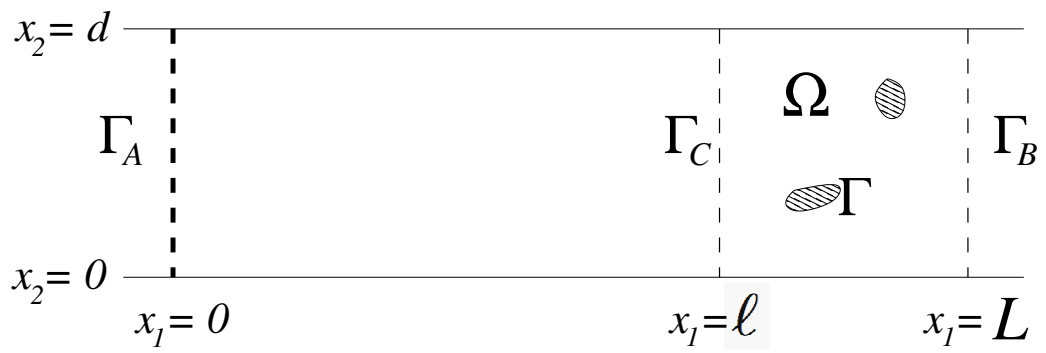

Figure 1. The waveguide, the scatterers and the time reversal mirror.

Denote by $\Omega=\Omega_{\infty} \backslash \overline{\mathcal{O}}$ the exterior of the scatterers, $\Gamma_{B}=\left\{x_{1}=L, 0<x_{2}<d\right\}$ and $\Gamma_{C}=\left\{x_{1}=\ell, 0<x_{2}<d\right\}$.

Throughout the paper, we assume that the time reversal mirror is located far enough from the scatterers (i.e. that $\ell$ is large enough). Under this assumption, a realistic model for the time reversal mirror is based on the emission and the reception of the propagating modes of the waveguide. More precisely, we assume that during the emission step the mirror generates an incident wave $u^{I}$ which is a superposition of guided modes of the waveguide propagating towards the scatterers:

$$
u^{I}(x)=\sum_{m=1}^{N} a_{m}^{I} \Phi_{m}^{+}(x), \quad \forall x=\left(x_{1}, x_{2}\right) \in \Omega_{\infty} .
$$

The vector $\mathbf{X}_{I}=\left(a_{1}^{I}, \cdots, a_{N}^{I}\right)^{\mathbf{T}} \in \mathbb{C}^{N}$, where

$$
a_{n}^{I}=\left(\left.u^{I}\right|_{\Gamma_{A}}, \phi_{n}\right)
$$

is the vector describing the "contribution" of each guided mode to the incident field. We define the emission operator as the operator $\mathcal{K}_{E}: \mathbf{X}_{I} \in \mathbb{C}^{N} \longmapsto u^{I} \in H_{\ell o c}^{1}\left(\Omega_{\infty}\right)$, where $u^{I}$ is given by (2.5). The diffracted field $u^{D}$ is the outgoing solution of the following boundary value problem set in $\Omega$ :

$$
\left\{\begin{array}{l}
\Delta u^{D}+k^{2} u^{D}=0 \quad \text { in } \Omega \\
u^{D}=-u^{I} \text { on } \Gamma \\
u^{D}=0 \text { on } \partial \Omega_{\infty}
\end{array}\right.
$$

Here, the outgoing behavior of the diffracted field is imposed by assuming that there exists complex coefficients $\left(a_{m}^{D}\right)_{m \geq 1}$ and $\left(b_{m}^{D}\right)_{m \geq 1}$ such that

$$
\begin{cases}\text { For } x_{1}<0: & u^{D}(x)=\sum_{m \in \mathbb{N}^{*}} a_{m}^{D} \Phi_{m}^{-}(x), \\ \text { For } x_{1}>L: & u^{D}(x)=\sum_{m \in \mathbb{N}^{*}} b_{m}^{D} \Phi_{m}^{+}(x) .\end{cases}
$$

In other words, an outgoing wave denotes a wave that can be decomposed as $\left|x_{1}\right| \rightarrow+\infty$ into a superposition of guided or evanescent modes. A rigorous justification of this definition can be obtained by proving a limiting absorption result, which is beyond the scope of the present paper.

We assume that the scattering problem (2.6) admits a unique solution. 
Remark 2.2. It is well known that the above problem is of Fredholm type. The uniqueness of its solution, and thus its existence, is also known to hold except for an at most countable family of singular values of the wavenumber $k$. In the case of a single convex scatterer, it can be proved that the set of these singular frequencies is empty (see for instance [17] where the case of a three dimensional waveguide is considered). Nevertheless, some configurations of non uniqueness (trapped modes) have been exhibited in the literature (cf. [10]), in particular in the case of Neumann boundary conditions and symmetric scatterers (of arbitrary shape) about the centerline of the waveguide.

We define the diffraction operator $\mathcal{K}_{D}: u^{I} \in H_{\ell o c}^{1}\left(\Omega_{\infty}\right) \longmapsto u^{D} \in H_{\ell o c}^{1}(\Omega)$, where $u^{D}$ is the unique solution of (2.6). Let us introduce the decomposition of the trace $u^{D}{ }_{\mid \Gamma_{A}}$ in the orthonormal basis $\left(\phi_{m}\right)_{m \in \mathbb{N}^{*}}$ of $L^{2}(0, d)$ :

$$
u_{\mid \Gamma_{A}}^{D}\left(x_{2}\right)=\sum_{m \in \mathbb{N}^{*}} a_{m}^{D} \phi_{m}\left(x_{2}\right),
$$

where

$$
a_{m}^{D}=\left(u_{\mid \Gamma_{A}}^{D}, \phi_{m}\right) .
$$

We assume that the mirror measures the coefficients of the modal decomposition of the trace $\left.u^{D}\right|_{A}$ corresponding to the guided modes, i.e. the coefficients $a_{m}^{D}$ for $1 \leq m \leq N$ in (2.7). The reception operator $\mathcal{K}_{R}$ is therefore defined by $\mathcal{K}_{R}: u^{D} \in H_{\ell o c}^{1}(\Omega) \longmapsto \mathbf{X}_{D}=\left(a_{1}^{D}, \cdots, a_{N}^{D},\right)^{\mathbf{T}} \in \mathbb{C}^{N}$. We define the scattering operator $\mathcal{S} \in \mathcal{L}\left(\mathbb{C}^{N}, \mathbb{C}^{N}\right)$ as the linear operator associating to $\mathbf{X}_{I}=\left(a_{1}^{I}, \cdots, a_{N}^{I}\right)^{\mathbf{T}}$ the vector $\mathbf{X}_{D}=\left(a_{1}^{D}, \cdots, a_{N}^{D}\right)^{\mathbf{T}}$ so that

$$
\mathbf{X}_{D}=\mathcal{S}\left(\mathbf{X}_{I}\right)=\left(\mathcal{K}_{R} \circ \mathcal{K}_{D} \circ \mathcal{K}_{E}\right)\left(\mathbf{X}_{I}\right)
$$

The time reversal experiment corresponds to two cycles, each cycle involving an emission step, a reception step and conjugation step (since time reversal amounts to a phase conjugation in the time-harmonic case). The time reversal operator $\mathcal{T} \in \mathcal{L}\left(\mathbb{C}^{N}, \mathbb{C}^{N}\right)$ is thus defined as follows:

$$
\mathcal{T}(\mathbf{X})=\overline{\mathcal{S}(\overline{\mathcal{S}(\mathbf{X})})}, \quad \forall \mathbf{X} \in \mathbb{C}^{N}
$$

If $\mathbb{S}=\left(S_{m n}\right)_{1 \leq m, n \leq N}$ and $\mathbf{T}=\left(T_{m n}\right)_{1 \leq m, n \leq N}$ denote respectively the matrices representing the linear operators $\mathcal{S}$ and $\mathcal{T}$ in the canonical basis $\left(\mathbf{e}_{m}\right)_{1 \leq m \leq N}$ of $\mathbb{C}^{N}$, then we have

$$
\mathbb{T}=\overline{\mathbb{S}} \mathbb{S}
$$

Remark 2.3. Let $u_{n}^{D}$ be the diffracted field corresponding to the incident wave

$$
u_{n}^{I}(x)=\mathcal{K}_{E} \mathbf{e}_{n}(x)=\exp \left(i \beta_{n} x_{1}\right) \phi_{n}\left(x_{2}\right) .
$$

Then, the elements $S_{m n}$ of the column $n$ of $\mathbb{S}$ are exactly the $N$ first coefficients of the modal decomposition of the trace $\left.u_{n}^{D}\right|_{\Gamma_{A}}$ in the orthonormal basis $\left(\phi_{m}\right)_{m \in \mathbb{N}^{*}}$ of $L^{2}(0, d): S_{m n}=\left(u_{n \mid \Gamma_{A}}^{D}, \phi_{m}\right)$. 


\section{Properties of the scattering and time reversal matrices}

We start this section by recalling some classical results on the Green's function of the

Helmholtz operator in the two-dimensional waveguide $\Omega_{\infty}=\mathbb{R} \times(0, d)$. For more details, we refer to [8].

Proposition 3.1. For all $x=\left(x_{1}, x_{2}\right) \in \Omega_{\infty}$, let $G(x, \cdot)$ be the outgoing Green's function of $\Delta+k^{2}$ in $\Omega_{\infty}$ :

$$
\left\{\begin{array}{l}
\Delta G(x, \cdot)+k^{2} G(x, \cdot)=\delta_{x} \quad \text { in } \Omega_{\infty} \\
G(x, \cdot)=0 \text { on } \partial \Omega_{\infty} \\
G(x, \cdot) \text { outgoing. }
\end{array}\right.
$$

Then, $G$ admits the modal decomposition

$$
G(x, y)=\sum_{m \in \mathbb{N}^{*}} \frac{1}{2 i \beta_{m}} \exp \left(i \beta_{m}\left|x_{1}-y_{1}\right|\right) \phi_{m}\left(x_{2}\right) \phi_{m}\left(y_{2}\right)
$$

where the functions $\phi_{m}$ and the coefficients $\beta_{m}$ are respectively defined by (2.1) and $(2.3)$.

Remark 3.2. Note that $G$ can be decomposed into a propagating part and an evanescent one : $G=G^{\text {prop }}+G^{\text {evan }}$, where

$$
\begin{aligned}
& G^{\text {prop }}(x, y)=\sum_{m=1}^{N} \frac{1}{2 i \beta_{m}} \exp \left(i\left|\beta_{m}\right|\left|x_{1}-y_{1}\right|\right) \phi_{m}\left(x_{2}\right) \phi_{m}\left(y_{2}\right) \\
& G^{\text {evan }}(x, y)=\sum_{m=N+1}^{\infty} \frac{1}{2 i \beta_{m}} \exp \left(-\left|\beta_{m}\right|\left|x_{1}-y_{1}\right|\right) \phi_{m}\left(x_{2}\right) \phi_{m}\left(y_{2}\right) .
\end{aligned}
$$

We also recall the following classical integral representation formula: given a bounded domain $\mathcal{U}$ contained in $\Omega_{\infty}$ and $u$ satisfying

$$
\left\{\begin{array}{l}
\Delta u+k^{2} u=0 \text { in } \Omega_{\infty} \backslash \overline{\mathcal{U}}, \\
u=0 \text { on } \partial \Omega_{\infty}, \\
u \text { outgoing. }
\end{array}\right.
$$

then, if $\nu$ denotes the unit outgoing normal to $\Omega_{\infty} \backslash \overline{\mathcal{U}}$, we have

$$
u(x)=\int_{\partial \mathcal{U}}\left(\frac{\partial G}{\partial \nu_{y}}(x, y) u(y)-G(x, y) \frac{\partial u}{\partial \nu}(y)\right) d y, \quad \forall x \in \Omega_{\infty} \backslash \overline{\mathcal{U}} .
$$

Using these preliminary results, we can prove the next Proposition which provides an integral representation of the coefficients of the scattering matrix $\mathbb{S}$.

Proposition 3.3. The coefficients $\left(S_{m n}\right)_{1 \leq m, n \leq N}$ of the scattering matrix $\mathbb{S}$ are given by the formula

$$
S_{m n}=-\int_{\Gamma} G_{m}(y) \frac{\partial u_{n}^{T}}{\partial \nu}(y) d y, \quad \forall 1 \leq m, n \leq N
$$

where $u_{n}^{T}=u_{n}^{I}+u_{n}^{D}$ is the total field corresponding to the incident wave

$$
u_{n}^{I}(x)=\mathcal{K}_{E} \mathbf{e}_{n}(x)=\exp \left(i \beta_{n} x_{1}\right) \phi_{n}\left(x_{2}\right)
$$

and where

$$
G_{m}(y)=\left(G(\cdot, y)_{\mid \Gamma_{A}}, \phi_{m}\right)=\frac{1}{2 i \beta_{m}} \exp \left(i \beta_{m} y_{1}\right) \phi_{m}\left(y_{2}\right)
$$


Proof. Fix some integer $n$ in $\{1, \cdots, N\}$ and some point $x \in \Omega_{\infty} \backslash \overline{\mathcal{O}}$. Since $\Delta u_{n}^{I}+k^{2} u_{n}^{I}=\Delta G(x, \cdot)+k^{2} G(x, \cdot)=0$ in $\mathcal{O}$, Green's formula shows that

$$
0=\int_{\Gamma}\left(\frac{\partial G}{\partial \nu_{y}}(x, y) u_{n}^{I}(y)-G(x, y) \frac{\partial u_{n}^{I}}{\partial \nu}(y)\right) d y, \quad \forall x \in \Omega_{\infty} \backslash \overline{\mathcal{O}} .
$$

On the other hand, by applying the integral representation (3.2) to the diffracted field $u_{n}^{D}$ corresponding to $u_{n}^{I}$, we obtain that for all $x \in \Omega_{\infty} \backslash \overline{\mathcal{O}}$, there holds

$$
u_{n}^{D}(x)=\int_{\Gamma}\left(\frac{\partial G}{\partial \nu_{y}}(x, y) u_{n}^{D}(y)-G(x, y) \frac{\partial u_{n}^{D}}{\partial \nu}(y)\right) d y
$$

By adding the last two relations and using the boundary condition $u_{n}^{T}=u_{n}^{I}+u_{n}^{D}=0$ on $\Gamma$, we obtain that

$$
u_{n}^{D}(x)=-\int_{\Gamma} G(x, y) \frac{\partial u_{n}^{T}}{\partial \nu}(y) d y .
$$

Since the coefficients $S_{m n}$, for $1 \leq m \leq N$, are nothing but the coefficients of the modal decomposition of the trace $\left.u_{n}^{D}\right|_{\Gamma_{A}}$ (see Remark 2.3), relation (3.6) shows that they can be derived from the corresponding coeffcients $\left(G_{m}(y)\right)_{1 \leq m \leq N}$ of $G(\cdot, y)$ and relation (3.3) is thus proved. The expression (3.5) of the coefficents $\left(G_{m}(y)\right)_{1 \leq m \leq N}$ follows then from (3.1).

In the following Lemma, we prove a symmetry property for the scattering matrix.

Lemma 3.4. Assume that $\mathbb{C}^{N}$ is endowed with the inner product

$$
\langle\mathbf{X}, \mathbf{Y}\rangle_{\beta}=\sum_{m=1}^{N} \beta_{m} X_{m} \overline{Y_{m}}
$$

where the positive constants $\beta_{m}$, for $1 \leq m \leq N$, are defined by (2.3). Then, the scattering matrix $\mathbb{S}$ satisfies

$$
\langle\mathbf{X}, \overline{\mathbb{S}} \mathbf{Y}\rangle_{\beta}=\langle\mathbb{S} X, \mathbf{Y}\rangle_{\beta}, \quad \forall \mathbf{X}, \mathbf{Y} \in \mathbb{C}^{N}
$$

Proof. Let us start by introducing some additional notation. Set

$$
\mathbf{X}=\left(a_{1}^{I}, \cdots, a_{N}^{I}\right)^{\mathbf{T}}, \quad \mathbf{Y}=\left(A_{1}^{I}, \cdots, A_{N}^{I}\right)^{\mathbf{T}} .
$$

Let $u^{I}=\mathcal{K}_{E} \mathbf{X}$ (respectively $v^{I}=\mathcal{K}_{E} \mathbf{Y}$ ) denote the incident field generated by $\mathbf{X}$ (respectively $\mathbf{Y}$ ), and let $u^{D}=\mathcal{K}_{D} u^{I}$ (respectively $v^{D}=\mathcal{K}_{D} v^{I}$ ) be the corresponding diffracted field. The associated total fields are then defined by

$$
u^{T}=u^{I}+u^{D}, \quad v^{T}=v^{I}+v^{D} .
$$

We also introduce the corresponding measurements $\mathbf{X}_{D}, \mathbf{Y}_{D} \in \mathbb{C}^{N}$ defined by:

$$
\mathbf{X}_{D}=\left(a_{1}^{D}, \cdots, a_{N}^{D}\right)^{\mathbf{T}}=\mathbb{S} \mathbf{X}, \quad \mathbf{Y}_{D}=\left(A_{1}^{D}, \cdots, A_{N}^{D}\right)^{\mathbf{T}}=\mathbb{S} \mathbf{Y} .
$$

Since $u^{T}=v^{T}=0$ on the boundary $\Gamma$ of the scatterers, we obviously have:

$$
\int_{\Gamma}\left(\frac{\partial u^{T}}{\partial \nu} v^{T}-u^{T} \frac{\partial v^{T}}{\partial \nu}\right)=0
$$


Inserting (3.9) into the above equation and setting $\mathcal{R}^{\alpha \beta}=\int_{\Gamma}\left(\frac{\partial u^{\alpha}}{\partial \nu} v^{\beta}-u^{\alpha} \frac{\partial v^{\beta}}{\partial \nu}\right)$ for $\alpha, \beta \in\{I, D\}$, we obtain that

$$
\mathcal{R}^{I I}+\mathcal{R}^{D D}+\mathcal{R}^{I D}+\mathcal{R}^{D I}=0,
$$

Since $u^{I}$ and $v^{I}$ satisfy the Helmholtz equation inside the scatterers, we have

$$
\mathcal{R}^{I I}=0 .
$$

Let us show that

$$
\mathcal{R}^{D D}=0 .
$$

We apply Green's formula to $u^{D}$ and $v^{D}$, which satisfy the Helmholtz equation in the domain $\mathcal{U}=\left\{x \in \Omega ; 0<x_{1}<L\right\}$ with boundary $\partial \mathcal{U}=\Gamma_{0} \cup \Gamma \cup \Gamma_{A} \cup \Gamma_{B}$, where $\Gamma_{0}=\left\{x=\left(x_{1}, 0\right) ; 0<x_{1}<L\right\} \cup\left\{x=\left(x_{1}, d\right) ; 0<x_{1}<L\right\}$. Since $u^{D}$ and $v^{D}$ vanish on $\Gamma_{0}$, we obtain

$$
\mathcal{R}^{D D}=-\int_{\Gamma_{A} \cup \Gamma_{B}}\left(\frac{\partial u^{D}}{\partial \nu} v^{D}-u^{D} \frac{\partial v^{D}}{\partial \nu}\right)=-\mathcal{J}_{A}\left(u^{D}, v^{D}\right)-\mathcal{J}_{B}\left(u^{D}, v^{D}\right),
$$

where we have set

$$
\mathcal{J}_{\gamma}\left(u^{\alpha}, v^{\beta}\right)=\int_{\Gamma_{\gamma}}\left(\frac{\partial u^{\alpha}}{\partial \nu} v^{\beta}-u^{\alpha} \frac{\partial v^{\beta}}{\partial \nu}\right), \quad \alpha, \beta \in\{I, D\}, \gamma \in\{A, B\} .
$$

If we introduce the modal decompositions of the traces $u^{D}{ }_{\mid \Gamma_{A}}$ in the orthonormal basis $\left(\phi_{m}\right)_{m \in \mathbb{N}^{*}}$ of $L^{2}(0, d): u^{D}{ }_{\mid \Gamma_{A}}\left(x_{2}\right)=\sum_{m \in \mathbb{N}^{*}} a_{m}^{D} \phi_{m}\left(x_{2}\right)$, then one can easily check, since $u^{D}$ is outgoing, that

$$
u^{D}(x)=\sum_{m \in \mathbb{N}^{*}} a_{m}^{D} \exp \left(-i \beta_{m} x_{1}\right) \phi_{m}\left(x_{2}\right), \quad \forall x_{1}<0 .
$$

Therefore, we have

$$
\left.\frac{\partial u^{D}}{\partial \nu}\right|_{\Gamma_{A}}=\sum_{m \in \mathbb{N}^{*}} i \beta_{m} a_{m}^{D} \phi_{m}=\mathcal{L}\left(u^{D}{ }_{\mid \Gamma_{A}}\right)
$$

where $\mathcal{L}: H^{1 / 2}(0, d) \longrightarrow H^{-1 / 2}(0, d)$ denotes the Dirichlet-to-Neumann operator:

$$
\mathcal{L}\left(\sum_{m \in \mathbb{N}^{*}} a_{m} \phi_{m}\right)=\sum_{m \in \mathbb{N}^{*}} i \beta_{m} a_{m} \phi_{m} .
$$

In a similar way, if

$$
v^{D}{ }_{\mid \Gamma_{A}}=\sum_{m \in \mathbb{N}^{*}} A_{m}^{D} \phi_{m},
$$

then

$$
\left.\frac{\partial v^{D}}{\partial \nu}\right|_{\Gamma_{A}}=\sum_{m \in \mathbb{N}^{*}} i \beta_{m} A_{m}^{D} \phi_{m} .
$$


Consequently, we have $\mathcal{J}_{A}\left(u^{D}, v^{D}\right)=\sum_{m \in \mathbb{N}^{*}}\left(i \beta_{m} a_{m}^{D} A_{m}^{D}-i \beta_{m} a_{m}^{D} A_{m}^{D}\right)=0$. One can show similarly that $\mathcal{J}_{B}\left(u^{D}, v^{D}\right)=0$ and we finally get (3.12) by using (3.13).

Let us now deal with the terms $\mathcal{R}^{I D}$ and $\mathcal{R}^{D I}$. Since $u^{I}$ and $v^{D}$ satisfy the Helmholtz equation in $\mathcal{U}$ and vanish on $\Gamma_{0}$, we have:

$$
\mathcal{R}^{I D}=-\mathcal{J}_{A}\left(u^{I}, v^{D}\right)-\mathcal{J}_{B}\left(u^{I}, v^{D}\right) .
$$

Let us consider first the term $\mathcal{J}_{A}\left(u^{I}, v^{D}\right)$. The definition (2.5) of $u^{I}$ shows that

$$
\left.\frac{\partial u^{I}}{\partial \nu}\right|_{\Gamma_{A}}=\sum_{m=1}^{N}\left(-i \beta_{m}\right) a_{m}^{I} \phi_{m} .
$$

Using for $\left.v^{D}\right|_{\Gamma_{A}}$ and $\left.\frac{\partial v^{D}}{\partial \nu}\right|_{\Gamma_{A}}$ the modal decompositions (3.15) and (3.16), we get that

$$
\mathcal{J}_{A}\left(u^{I}, v^{D}\right)=-\sum_{m=1}^{N} 2 i \beta_{m} a_{m}^{I} A_{m}^{D} .
$$

Similar computations show that the term corresponding to $\Gamma_{B}$ is

$$
\mathcal{J}_{B}\left(u^{I}, v^{D}\right)=0 .
$$

Relations (3.17), (3.18) and (3.19) imply that

$$
\mathcal{R}^{I D}=\sum_{m=1}^{N} 2 i \beta_{m} a_{m}^{I} A_{m}^{D}
$$

By symmetry, we have

$$
\mathcal{R}^{D I}=-\sum_{m=1}^{N} 2 i \beta_{m} A_{m}^{I} a_{m}^{D} .
$$

By adding relations (3.11), (3.12), (3.20) and (3.21), relation (3.10) yields $\sum_{m=1}^{N} \beta_{m} a_{m}^{I} A_{m}^{D}=\sum_{m=1}^{N} \beta_{m} A_{m}^{I} a_{m}^{D}$, or equivalently

$$
\left\langle\mathbf{X}, \overline{\mathbf{Y}_{D}}\right\rangle_{\beta}=\left\langle\mathbf{X}_{D}, \overline{\mathbf{Y}}\right\rangle_{\beta}
$$

Since $\mathbf{X}_{D}=\mathbb{S} \mathbf{X}$ and $\mathbf{Y}_{D}=\mathbb{S} \mathbf{Y}$, the above relation shows that $\langle\mathbf{X}, \overline{\mathbb{S}} \overline{\mathbf{Y}}\rangle_{\beta}=\langle\mathbb{S} \mathbf{X}, \overline{\mathbf{Y}}\rangle_{\beta}$ for all $\mathbf{X}, \mathbf{Y} \in \mathbb{C}^{N}$ and the proof is thus complete.

Let $\left(\widehat{\mathbf{e}}_{\mathbf{m}}\right)_{1 \leq m \leq N}$ be the following orthonormal basis of $\mathbb{C}^{N}$ for the scalar product $\langle\cdot, \cdot\rangle_{\beta}$ defined by $(3.7)$ :

$$
\widehat{\mathbf{e}}_{\mathbf{m}}=\frac{1}{\sqrt{\beta_{m}}} \mathbf{e}_{\mathbf{m}}, \quad \forall 1 \leq m \leq N .
$$

Lemma 3.4 implies then the following result. 
Proposition 3.5. Let $\widehat{\mathbb{S}}=\left(\widehat{S}_{m n}\right)$ denote the matrix representing the scattering operator $\mathcal{S}$ in the orthonormal basis $\left(\widehat{\mathbf{e}}_{\mathbf{m}}\right)_{1 \leq m \leq N}$ of $\mathbb{C}^{N}$ defined by (3.22). Then, $\widehat{\mathbb{S}}$ is a symmetric matrix, and thus

$$
\widehat{\mathbb{S}}^{*}=\widehat{\widehat{\mathbb{S}}}
$$

Consequently, if $\widehat{\mathbb{T}}$ denotes the matrix representing $\mathcal{T}$ in the orthonormal basis $\left(\widehat{\mathbf{e}}_{\mathbf{m}}\right)_{1 \leq m \leq N}$, then we have

$$
\widehat{\mathbb{T}}=\overline{\mathbb{S}} \widehat{\mathbb{S}}=\widehat{\mathbb{S}} * \widehat{\mathbb{S}}
$$

Proof. By applying relation (3.8) with $\mathbf{X}=\mathbf{e}_{\mathbf{m}}$ and $\mathbf{Y}=\mathbf{e}_{\mathbf{n}}$, we obtain that

$$
\beta_{m} S_{m n}=\beta_{n} S_{n m}, \quad \forall 1 \leq m, n \leq N .
$$

Since $\widehat{S}_{m n}=\sqrt{\beta_{m} / \beta_{n}} S_{m n}$, the above relation implies (3.23).

Proposition 3.5 shows that $\widehat{\mathbb{T}}$ is hermitian and positive. In particular, the eigenvalues of $\mathbb{T}$ are thus real and nonnegative.

\section{Selective focusing}

This Section is devoted to the asymptotic analysis of the eigenelements of $\mathbb{T}$ as the size of the scatterers tends to 0 . These asymptotic results provide the key ingredient for studying the selective focusing properties of the D.O.R.T. method. From now on, we assume that the scatterers contained in the waveguide constitute a family $\left\{\mathcal{O}_{p}^{\varepsilon} ; 1 \leq p \leq M\right\}$ depending on a small parameter $\varepsilon$, where each obstacle $\mathcal{O}_{p}^{\varepsilon}$ is obtained from a reference open obstacle $\mathcal{O}_{p}$ (which is assumed to contain the origin) by a dilation of ratio $\varepsilon$ centered at the point $s_{p}=\left(s_{p}^{1}, s_{p}^{2}\right) \in \mathbb{R}^{2}$ :

$$
\mathcal{O}_{p}^{\varepsilon}=\left\{x \in \mathbb{R}^{2} ; \xi=\left(x-s_{p}\right) / \varepsilon \in \mathcal{O}_{p}\right\} .
$$

We denote by $\Gamma_{p}^{\varepsilon}$ (respectively $\Gamma_{p}$ ) the boundary of $\mathcal{O}_{p}^{\varepsilon}$ (respectively $\mathcal{O}_{p}$ ), and we set $\Gamma^{\varepsilon}=\bigcup_{1 \leq p \leq M} \Gamma_{p}^{\varepsilon}$ and $\mathcal{O}^{\varepsilon}=\bigcup_{1 \leq p \leq M} \mathcal{O}_{p}^{\varepsilon}$.

In Subsection 4.1, we first obtain the asymptotics of the scattering matrix $\mathbb{S}^{\varepsilon}$ and thus of the time reversal $\mathbb{T}^{\varepsilon}$ as the size of the scatterers $\varepsilon \rightarrow 0$. Next, we prove in Subsection 4.2 a technical result (Proposition 4.3) that is used in Subsection 4.3 to obtain an approximation of the eigenvalues and eigenvectors of the limit time reversal matrix as the number $N$ of guided modes of the waveguide tends to $+\infty$.

\subsection{Asymptotic analysis}

The behavior of the scattering matrix $\mathbb{S}^{\varepsilon}$ for small scatterers is provided by the following result.

Proposition 4.1. There exist $M$ positive constants $\kappa_{1}, \ldots, \kappa_{M}$ depending only on the geometry of the reference obstacles $\mathcal{O}_{1}, \ldots, \mathcal{O}_{M}$ such that the scattering matrix $\mathbb{S}^{\varepsilon}$ satisfies

$$
\mathbb{S}^{\varepsilon}=\mathbb{S}^{0}+\mathcal{O}\left(\frac{1}{\ln \mathrm{e}}\right)=\left(\sum_{p=1}^{M} \mathbb{S}^{0, p}\right)+\mathcal{O}\left(\frac{1}{\ln \mathrm{e}}\right),
$$


where each matrix $\mathbb{S}^{0, p}=\left(S_{m n}^{0, p}\right)_{1 \leq m, n \leq N}$ is defined by

$$
\mathbb{S}^{0, p}=\kappa_{p} \mathbf{U}_{p} \mathbf{V}_{p}^{\mathbf{T}} \quad \forall 1 \leq p \leq M,
$$

where the vectors $\mathbf{U}_{p}=\left(U_{m}^{p}\right), \mathbf{V}_{p}=\left(V_{m}^{p}\right) \in \mathbb{C}^{N}$ are defined by:

$$
\left\{\begin{array}{l}
U_{m}^{p}=G_{m}\left(s_{p}\right)=\frac{1}{2 i \beta_{m}} \exp \left(i \beta_{m} s_{p}^{1}\right) \phi_{m}\left(s_{p}^{2}\right), \\
V_{m}^{p}=u_{m}^{I}\left(s_{p}\right)=\Phi_{m}^{+}\left(s_{p}\right)=\exp \left(i \beta_{m} s_{p}^{1}\right) \phi_{m}\left(s_{p}^{2}\right),
\end{array}\right.
$$

Proof. According to Proposition 3.3, the coeffcient $S_{m n}^{\varepsilon}$ of $\mathbb{S}^{\varepsilon}$ reads for all $1 \leq m, n \leq N$ :

$$
S_{m n}^{\varepsilon}=-\int_{\Gamma^{\varepsilon}} G_{m}(y)\left(\frac{\partial u_{n}^{I}}{\partial \nu}(y)+\frac{\partial u_{\varepsilon, n}^{D}}{\partial \nu}(y)\right) d y=\sum_{p=1}^{M} S_{m n}^{\varepsilon, p},
$$

where we have set

$$
S_{m n}^{\varepsilon, p}=-\int_{\Gamma_{p}^{\varepsilon}} G_{m}(y)\left(\frac{\partial u_{n}^{I}}{\partial \nu}(y)+\frac{\partial u_{\varepsilon, n}^{D}}{\partial \nu}(y)\right) d y .
$$

Since the integral involving the incident field in the above expression is of order $\varepsilon$, the change of variable $y=\varepsilon \xi+s_{p}$ shows that

$$
S_{m n}^{\varepsilon, p}=-\int_{\Gamma_{p}} G_{m}\left(\varepsilon \xi+s_{p}\right) \frac{\partial \varphi_{p}^{\varepsilon, n}}{\partial \nu}(\xi) d \xi+\mathcal{O}(\varepsilon),
$$

where we have set $\varphi_{p}^{\varepsilon, n}(\xi)=u_{\varepsilon, n}^{D}(x)$ for all $\xi=\left(x-s_{p}\right) / \varepsilon \in \mathcal{O}_{p}$. The next step consists in obtaining the asymptotic of $\varphi_{p}^{\varepsilon, n}$. This is achieved in Proposition 6.1 proved in the Appendix. According to this result, we have

$$
\left\|\varphi_{p}^{\varepsilon, n}+u_{n}^{I}\left(s_{p}\right) \Theta_{p}\right\|_{H^{1}\left(D_{p}\right)}=\mathcal{O}\left(\frac{1}{\ln \mathrm{e}}\right)
$$

where $D_{p}$ is a neighborhood of $\Gamma_{p}$ and $\Theta_{p}$ denotes the static potential associated to the obstacle $p$, i.e.

$$
\left\{\begin{array}{l}
\Delta \Theta_{p}=0 \text { in } \mathbb{R}^{2} \backslash \overline{\mathcal{O}_{p}} \\
\Theta_{p}=1 \text { on } \Gamma_{p}, \\
\Theta_{p}(x)=\mathcal{O}(1), \text { for }|x| \rightarrow+\infty
\end{array}\right.
$$

Using the asymptotic expansion (4.3) in (4.2) yields

$$
S_{m n}^{\varepsilon, p}=G_{m}\left(s_{p}\right) u_{n}^{I}\left(s_{p}\right) \int_{\Gamma_{p}} \frac{\partial \Theta_{p}}{\partial \nu}(\xi) d \xi+\mathcal{O}\left(\frac{1}{\ln \mathrm{e}}\right)=S_{m n}^{0, p}+\mathcal{O}\left(\frac{1}{\ln \mathrm{e}}\right) .
$$

Thus $\mathbb{S}^{\varepsilon}=\mathbb{S}^{0}+\mathcal{O}\left(\frac{1}{\ln \mathrm{e}}\right)$ where the coefficient $S_{m n}^{\varepsilon}$ of the limit scattering matrix $\mathbb{S}^{0}$ is given by

$$
S_{m n}^{0}=\sum_{p=1}^{M} S_{m n}^{0, p}, \quad S_{m n}^{0, p}=\kappa_{p} G_{m}\left(s_{p}\right) u_{n}^{I}\left(s_{p}\right)=\kappa_{p} U_{m}^{p} V_{n}^{p},
$$


and where $\kappa_{p}=\int_{\Gamma_{p}} \frac{\partial \Theta_{p}}{\partial \nu}=\int_{\mathbb{R}^{2} \backslash \mathcal{O}_{p}}\left|\nabla \Theta_{p}\right|^{2}$ denotes the capacity of the scatterer $p$.

Thanks to the definition (2.9) of the time reversal matrix, the above result implies immediately the following one.

Corollary 4.2. With the notation of Proposition 4.1, the time reversal matrix $\mathbb{T}^{\varepsilon}$ admits the following asymptotics for small scatterers:

$$
\mathbb{T}^{\varepsilon}=\mathbb{T}^{0}+\mathcal{O}\left(\frac{1}{\ln \mathrm{e}}\right)
$$

in which

$$
\mathbb{T}^{0}=\sum_{p=1}^{M} \sum_{q=1}^{M} \kappa_{p} \kappa_{q} \quad\left(\mathbf{V}_{q}^{*} \mathbf{U}_{p}\right) \overline{\mathbf{U}_{q}} \mathbf{V}_{p}^{\mathbf{T}},
$$

where the star denotes the conjugate transpose.

\subsection{A preliminary result}

This Subsection is devoted to the proof of a technical result constituting one of the main ingredients used in Subsection 4.3 to establish the selective focusing properties of the D.O.R.T method. We first introduce some additional notation. Let $\left(\tau_{N}\right)_{N \in \mathbb{N}^{*}}$ be a sequence of real numbers such that there exists $0<\delta^{*}<1$ satisfying

$$
N+\delta^{*}<\tau_{N}<N+1, \quad \forall N \geq 1 .
$$

Note that condition (4.5) implies in particular that $\tau_{N} \notin \mathbb{N}^{*}$ and that

$$
N=\left\lfloor\tau_{N}\right\rfloor
$$

Finally, set

$$
\beta_{m}^{N}=\sqrt{\tau_{N}^{2}-m^{2}}, \quad \forall m=1, \ldots, N
$$

and define for all $\left(X_{1}, X_{2}\right) \in \mathbb{R} \times[-\pi, \pi]$ :

$$
\rho_{N}\left(X_{1}, X_{2}\right)=\sum_{m=1}^{N} \frac{1}{\beta_{m}^{N}} \exp \left[i\left(\beta_{m}^{N} X_{1}+m X_{2}\right)\right] .
$$

The next Proposition provides the behavior of $\rho_{N}$ as $N \rightarrow \infty$.

Proposition 4.3. With the notation above, let $\theta \in(0,1], \eta>0$ and let $I$ be a bounded interval of $\mathbb{R}$. Then, under the assumption (4.5), $\rho_{N}\left(X_{1}, X_{2}\right)$ satisfies the following properties:

i) There exists $\alpha>0$ such that

$$
\lim _{N \rightarrow+\infty} \rho_{N}(0,0)=\alpha .
$$

ii) As $N \rightarrow+\infty$, the estimate

$$
\rho_{N}\left(0, X_{2}\right)=\mathcal{O}\left(N^{-\theta / 2}\right)
$$


Time reversal in acoustic waveguides

holds uniformly for $X_{2} \in[-\pi, \pi]$ satisfying

$$
\left|X_{2}\right| \geq \eta N^{(\theta-1) / 2}
$$

iii) As $N \rightarrow+\infty$, the following estimate

$$
\rho_{N}\left(X_{1}, X_{2}\right)=\mathcal{O}\left(N^{-1 / 4}\right)+\mathcal{O}\left(N^{-\theta / 2}\right)
$$

holds uniformly for $X=\left(X_{1}, X_{2}\right) \in I \times[-\pi, \pi]$ satisfying

$$
\left|X_{1}\right| \geq \eta N^{\theta-1} \text {. }
$$

Proof. i) We have

$$
\rho_{N}(0,0)=\sum_{m=1}^{N} 1 / \beta_{m}^{N}=\sum_{m=1}^{N}\left(\tau_{N}^{2}-m^{2}\right)^{-1 / 2}
$$

Since $\lim _{N \rightarrow+\infty} \sum_{m=1}^{N-1}\left(N^{2}-m^{2}\right)^{-1 / 2}=\pi / 2$ and since $N=\left\lfloor\tau_{N}\right\rfloor$, the sum $\rho_{N}(0,0)$ admits a limit $\alpha>0$.

In order to prove assertions $i i$ ) and $i i i)$, we first rearrange the terms of the sum $\rho_{N}\left(X_{1}, X_{2}\right)$ by setting $n=N-m$. We obtain that

$$
\rho_{N}\left(X_{1}, X_{2}\right)=\sum_{n=0}^{N-1} \frac{\exp \left(i f_{N}(n)\right)}{g_{N}(n)}
$$

where we have set

$$
f_{N}(n)=X_{1} g_{N}(n)+X_{2}(N-n) .
$$

and

$$
g_{N}(n)=\beta_{N-n}^{N}=\left[\tau_{N}^{2}-(N-n)^{2}\right]^{1 / 2}=\left[\tau_{N}^{2}-N^{2}+n(2 N-n)\right]^{1 / 2} .
$$

ii) We have

$$
\left|\rho_{N}\left(0, X_{2}\right)\right|=\left|\sum_{n=0}^{N-1} \frac{\exp \left(i n X_{2}\right)}{g_{N}(n)}\right|
$$

If we set, for $X_{2} \neq 0$,

$$
F_{n}=\sum_{k=0}^{n-1} \exp \left(i k X_{2}\right)=\frac{\sin \left(n X_{2} / 2\right)}{\sin \left(X_{2} / 2\right)} \exp \left[i(n-1) X_{2} / 2\right], \quad \forall n \in \mathbb{N}^{*},
$$

and $F_{0}=0$, then (4.14) implies that $\left|\rho_{N}\left(0, X_{2}\right)\right|=\left|\sum_{n=0}^{N-1} \frac{\left(F_{n+1}-F_{n}\right)}{g_{N}(n)}\right|$, and thus

$$
\left|\rho_{N}\left(0, X_{2}\right)\right|=\left|\frac{F_{N}}{g_{N}(N-1)}+\sum_{n=1}^{N-1} F_{n} D_{N}(n)\right|,
$$

where we have set $\left.D_{N}(n)=\left[g_{N}(n-1)\right)\right]^{-1}-\left[g_{N}(n)\right]^{-1}$. Relation (4.15) shows that for all $X_{2}$ satisfying (4.10), we have

$$
\left|F_{n}\right|=\mathcal{O}\left(N^{(1-\theta) / 2}\right) .
$$


Consequently, since $N=\left\lfloor\tau_{N}\right\rfloor$, we have

$$
\left|\frac{F_{N}}{g_{N}(N-1)}\right| \leq C \frac{N^{(1-\theta) / 2}}{\sqrt{\tau_{N}^{2}-1}}=\mathcal{O}\left(N^{-\theta / 2}\right) .
$$

On the other hand

$$
\begin{aligned}
D_{N}(n) & =\frac{g_{N}^{2}(n)-g_{N}^{2}(n-1)}{g_{N}(n-1) g_{N}(n)\left[g_{N}(n-1)+g_{N}(n)\right]}, \\
& =\frac{2(N-n)+1}{g_{N}(n-1) g_{N}(n)\left[g_{N}(n-1)+g_{N}(n)\right]}
\end{aligned}
$$

Using the fact that $x \rightarrow g_{N}(x)$ is nondecreasing for $x \in(0, N)$, the above relation yields

$$
\left|D_{N}(n)\right| \leq \frac{2(N-n)+1}{\left[g_{N}(n-1)\right]^{3}} .
$$

By noting that $g_{N}(n-1)=\left[\left(\tau_{N}^{2}-N^{2}\right)+(n-1)(2 N-n+1)\right]^{1 / 2}$, we obtain by using assumption (4.5) that for all $n=1, \cdots, N-1$ :

$$
\left[g_{N}(n-1)\right]^{2} \geq 2 \delta^{*} N+(n-1)(2 N-n) \geq\left(2 \delta^{*}+(n-1)\right) N .
$$

This last inequality and (4.19) yield then

$$
\left|D_{N}(n)\right| \leq \frac{2(N-n)+1}{\left(2 \delta^{*}+(n-1)\right)^{3 / 2} N^{3 / 2}} \leq \frac{4}{\left(2 \delta^{*}+(n-1)\right)^{3 / 2} N^{1 / 2}} .
$$

Now, (4.17) and (4.20) show that

$$
\left|\sum_{n=0}^{N-1} F_{n} D_{N}(n)\right|=\mathcal{O}\left(N^{-\theta / 2}\right) .
$$

Finally, the above inequality, (4.16) and (4.18) imply the desired relation (4.9).

iii) For all $N \in \mathbb{N}^{*}$ and all integer $n$ satisfying $1 \leq n \leq N$, let us set

$$
F(n, N)=\sum_{k=0}^{n-1} \exp \left[i f_{N}(k)\right],
$$

and $F(0, N)=0$. Then, equation (4.13) also reads

$$
\rho_{N}\left(X_{1}, X_{2}\right)=\sum_{n=0}^{N-1} \frac{F(n+1, N)-F(n, N)}{g_{N}(n)},
$$

or equivalently,

$$
\rho_{N}\left(X_{1}, X_{2}\right)=\frac{F(N, N)}{g_{N}(N-1)}+\sum_{n=0}^{N-1} F(n, N) D_{N}(n) .
$$


According to Lemma 4.4 proved below, we have for all $1 \leq n \leq N$ :

$$
|F(n, N)| \leq C N^{1 / 4}\left(n^{1 / 4}+n^{3 / 4} N^{-\theta / 2}\right) .
$$

The above inequality and (4.20) show that

$$
\begin{aligned}
\left|\sum_{n=0}^{N-1} F(n, N) D_{N}(n)\right| \leq C N^{-1 / 4}(1+ & \left.N^{-\theta / 2} \sum_{n=1}^{N} n^{-3 / 4}\right) \\
& =\mathcal{O}\left(N^{-1 / 4}\right)+\mathcal{O}\left(N^{-\theta / 2}\right) .
\end{aligned}
$$

On the other hand, once again by using Lemma 4.4, we have that

$$
|F(N, N)| \leq C\left(N^{1 / 2}+N^{1-\theta / 2}\right)=\mathcal{O}\left(N^{1-\theta / 2}\right),
$$

and thus

$$
\left|\frac{F(N, N)}{g_{N}(N-1)}\right| \leq C \frac{N^{1-\theta / 2}}{\sqrt{\tau_{N}^{2}-1}}=\mathcal{O}\left(N^{-\theta / 2}\right) .
$$

Relation (4.11) follows then from (4.21), (4.23) and the above relation.

Lemma 4.4. With the notation introduced at the beginning of this subsection, set for all $n \in\{1, \ldots, N\}$

$$
\left\{\begin{array}{l}
g_{N}(n)=\beta_{N-n}^{N}=\left[\tau_{N}^{2}-(N-n)^{2}\right]^{1 / 2} \\
f_{N}(n)=X_{1} g_{N}(n)+X_{2}(N-n), \\
F(n, N)=\sum_{k=0}^{n-1} \exp \left[i f_{N}(k)\right] .
\end{array}\right.
$$

Then, given $\theta \in(0,1], \eta>0$ and a bounded interval $I$ of $\mathbb{R}$, there exists $C>0$ such that for all $X_{1} \in I$ satisfying

$$
\left|X_{1}\right| \geq \eta N^{\theta-1}
$$

we have

$$
|F(n, N)| \leq C N^{1 / 4}\left(n^{1 / 4}+n^{3 / 4} N^{-\theta / 2}\right) .
$$

Proof. Set

$$
R_{n}=\max \left\{r \in \mathbb{N}^{*} \mid 1+2^{r} \leq n-1\right\},
$$

and write $F(n, N)$ as

$$
F(n, N)=\sum_{r=0}^{R_{n}} \sigma_{r}
$$

where we have set

$$
\sigma_{r}=\sum_{k=1+2^{r}}^{2^{r+1}} \exp \left[i f_{N}(k)\right] \quad \text { for } 1 \leq r \leq R_{n}-1,
$$

while $\sigma_{0}=\sum_{k=0}^{2} \exp \left[i f_{N}(k)\right]$ and $\sigma_{R_{n}}=\sum_{k=1+2^{R_{n}}}^{n-1} \exp \left[i f_{N}(k)\right]$.

According to Van der Corput Lemma (see Theorem 5 of $[18, \mathrm{p} .94]$ ), if $\left|f_{N}^{\prime \prime}(k)\right|$ is of 
order $\Lambda_{r}$ for $2^{r} \leq k \leq 2^{r+1}-1$, then each sum $\sigma_{r}$ of the form (4.27) can be estimated by

$$
\left|\sigma_{r}\right| \leq\left(1+2^{r}\right) \sqrt{\Lambda_{r}}+\frac{1}{\sqrt{\Lambda_{r}}} .
$$

But a straightforward computation shows that

$$
f_{N}^{\prime \prime}(k)=-X_{1} \tau_{N}^{2}\left[g_{N}(k)\right]^{-3 / 2} .
$$

In the rest of the proof, $C$ denotes a positive constant independent of $N$. Since $N=$ $\left\lfloor\tau_{N}\right\rfloor$ and $g_{N}(k)=\left[\tau_{N}^{2}-N^{2}+n(2 N-k)\right]^{1 / 2}$, we have $\left|f_{N}^{\prime \prime}(k)\right| \leq C\left|X_{1}\right| N^{2}(k N)^{-3 / 2}$ and thus

$$
\left|f_{N}^{\prime \prime}(k)\right| \leq C\left|X_{1}\right| N^{1 / 2} 2^{-3 r / 2}:=\Lambda_{r}, \quad \text { for } 2^{r}+1 \leq k \leq 2^{r+1} .
$$

Inserting the above estimate into inequality (4.28), we obtain thanks to (4.24) that

$$
\left|\sigma_{r}\right| \leq C N^{1 / 4}\left(2^{r / 4}+N^{-\theta / 2} 2^{3 r / 4}\right), \quad \forall 0 \leq r \leq R_{n}
$$

Summing the above inequalities for $0 \leq r \leq R_{n}$ yields

$$
|F(n, N)| \leq C N^{1 / 4}\left(2^{R_{n} / 4}+N^{-\theta / 2} 2^{3 R_{n} / 4}\right)
$$

The claimed estimate (4.25) follows immediately from the above inequality if we notice that from the definition (4.26) of $R_{n}$, we have $2^{R_{n}} \leq n$.

\subsection{Spectral analysis of the limit model for large values of $N$}

Let us start by recalling that according to Corollary 4.2 , the time reversal matrix $\mathbb{T}^{\varepsilon}$ admits the asymptotics

$$
\mathbb{T}^{\varepsilon}=\mathbb{T}^{0}+\mathcal{O}\left(\frac{1}{\ln \mathrm{e}}\right)
$$

where

$$
\mathbb{T}^{0}=\sum_{p=1}^{M} \sum_{q=1}^{M} \kappa_{p} \kappa_{q} \quad\left(\mathbf{V}_{q}^{*} \mathbf{U}_{p}\right) \overline{\mathbf{U}_{q}} \mathbf{V}_{p}^{\mathbf{T}}
$$

Clearly, the range of $\mathbb{T}^{0}$ satisfies $\operatorname{Ran}\left(\mathbb{T}^{0}\right) \subset<\overline{\mathbf{U}_{1}}, \ldots, \overline{\mathbf{U}_{M}}>$ and thus, the number of non zero eigenvalues of $\mathbb{T}^{0}$ is at most equal to the number of scatterers $M$. In this Subsection, our aim is twofold : first, to determine an approximation of the eigenvectors of $\mathbb{T}^{0}$ as the number of propagating modes $N=\lfloor k d / \pi\rfloor$ tends to $+\infty$ (note that this situation corresponds to the case where the wavelength $\lambda$ is small compared to the height of the waveguide : $\lambda<<d$ ). Secondly, to verify that these (approximate) eigenvectors generate incident waves that focus selectively on each scatterer. These two results are respectively provided by Proposition 4.5 and Proposition 4.7.

A technical assumption needed to establish these results is to suppose that the frequencies $k$ considered in our asymptotic analysis as $N \rightarrow+\infty$ (or equivalently $k d \rightarrow+\infty)$ remain away from the cutoff frequencies of the waveguide. More precisely, we assume that the following condition is satisfied:

$$
\exists \delta^{*}>0 \text { such that : } \frac{k d}{\pi}-N>\delta^{*} .
$$


In order to determine approximate eigenvectors of $\mathbb{T}^{0}$, let us note that the scalar products $\mathbf{V}_{q}^{*} \mathbf{U}_{p}$ are given by the formulas

$$
\mathbf{V}_{q}^{*} \mathbf{U}_{p}=\sum_{m=1}^{N} \frac{1}{2 i \beta_{m}} \exp \left[i \beta_{m}\left(s_{p}^{1}-s_{q}^{1}\right)\right] \phi_{m}\left(s_{p}^{2}\right) \phi_{m}\left(s_{q}^{2}\right) .
$$

Then, the following result holds true.

Proposition 4.5. Let $p \in\{1, \ldots, M\}$ and let $\alpha$ be defined by Proposition 4.3. Then, under assumption (4.30), we have

$$
\lim _{N \rightarrow+\infty} \mathbf{V}_{q}^{*} \mathbf{U}_{p}=\alpha /(2 i \pi) .
$$

Moreover, for all $q \in\{1, \ldots, M\}$, with $q \neq p$, the following estimate holds as $N \rightarrow+\infty:$

$$
\mathbf{V}_{q}^{*} \mathbf{U}_{p}= \begin{cases}\mathcal{O}\left(N^{-1 / 4}\right) & \text { if } s_{p}^{1} \neq s_{q}^{1} \\ \mathcal{O}\left(N^{-1 / 2}\right) & \text { if } s_{p}^{1}=s_{q}^{1}\end{cases}
$$

Proof. Let $q \in\{1, \ldots, M\}$. Formula (4.31) shows that

$$
\mathbf{V}_{q}^{*} \mathbf{U}_{p}=\frac{1}{i} \sum_{m=1}^{N} \frac{1}{d \beta_{m}} \exp \left[i \beta_{m}\left(s_{p}^{1}-s_{q}^{1}\right)\right] \sin \left(m \pi s_{p}^{2} / d\right) \sin \left(m \pi s_{q}^{2} / d\right) .
$$

Setting in the above expression $X_{1}=\pi\left(s_{p}^{1}-s_{q}^{1}\right) / d, X_{2}^{ \pm}=\pi\left(s_{p}^{2} \pm s_{q}^{2}\right) / d$ and $\beta_{m}^{N}=d \beta_{m} / \pi=\sqrt{(k d / \pi)^{2}-m^{2}}$, a straightforward computation shows that

$$
\begin{aligned}
\mathbf{V}_{q}^{*} \mathbf{U}_{p}=-\frac{1}{4 i \pi} \sum_{m=1}^{N} \frac{1}{\beta_{m}^{N}} \exp \left(i \beta_{m}^{N} X_{1}\right)\left[\exp \left(i m X_{2}^{+}\right)+\exp \left(-i m X_{2}^{+}\right)\right. \\
\left.-\exp \left(i m X_{2}^{-}\right)-\exp \left(i m X_{2}^{-}\right)\right] .
\end{aligned}
$$

Therefore, using the notation introduced in Subsection 4.2 and setting $\tau_{N}=k d / \pi$, we have

$$
\mathbf{V}_{q}^{*} \mathbf{U}_{p}=-\frac{1}{4 i \pi}\left[\rho_{N}\left(X_{1}, X_{2}^{+}\right)+\rho_{N}\left(X_{1},-X_{2}^{+}\right)-\rho_{N}\left(X_{1}, X_{2}^{-}\right)-\rho_{N}\left(X_{1},-X_{2}^{-}\right)\right] .
$$

In the particular case where $q=p$, the above relation reads

$$
\mathbf{V}_{p}^{*} \mathbf{U}_{p}=-\frac{1}{4 i \pi}\left[\rho_{N}\left(0, X_{2}^{+}\right)+\rho_{N}\left(0,-X_{2}^{+}\right)-2 \rho_{N}(0,0)\right]
$$

with $X_{2}^{+}=2 \pi s_{p}^{2} / d \in(0,2 \pi)$. Assertion $i$ ) of Proposition 4.3 (applied with $\theta=1$ ) implies that

$$
\lim _{N \rightarrow+\infty} \rho_{N}\left(0, X_{2}^{+}\right)=\lim _{N \rightarrow+\infty} \rho_{N}\left(0,-X_{2}^{+}\right)=0 .
$$

Therefore, by applying assertion $i$ ) in Proposition 4.3, we obtain relation (4.32). In the case $q \neq p$, we have $0<X_{2}^{+}<2 \pi$ and $-\pi<X_{2}^{-}<\pi$. The claimed estimate (4.33) follows then from assertion iii) of Proposition 4.3 (once again for the particular case $\theta=1)$ by using the fact that $X_{2} \mapsto \rho_{N}\left(X_{1}, X_{2}\right)$ is $2 \pi$-periodic. 
Remark 4.6. The result of Proposition 4.5 can be justified qualitatively as follows. First, note that for $s_{p}^{1}-s_{q}^{1} \geq 0$, we have $\mathbf{V}_{q}^{*} \mathbf{U}_{p}=G^{\text {prop }}\left(s_{p}, s_{q}\right)$, where $G^{\text {prop }}$ denotes the propagating part of the Green's function $G$ of the waveguide(see Remark 3.2). Similarly, for $s_{p}^{1}-s_{q}^{1}<0$, we have $\mathbf{V}_{q}^{*} \mathbf{U}_{p}=-\overline{G^{\text {prop }}\left(s_{p}, s_{q}\right)}$. Since $G^{\text {prop }}(x, y)$ behaves like $G(x, y)$ as the number of propagating modes $N$ tends to $+\infty$, and since $G(x, y)$ is singular for $x=y$, it follows that $G^{\text {prop }}(x, y)$ is small compared to $G^{\text {prop }}(x, x)$ for $N$ large enough, or equivalently, that $\mathbf{V}_{q}^{*} \mathbf{U}_{p}$ is small compared to $\mathbf{V}_{p}^{*} \mathbf{U}_{p}$ for $q \neq p$.

Now, let us note that according to (4.29), we have for all $p \in\{1, \ldots, M\}$ :

$$
\mathbb{T}^{0} \overline{\mathbf{U}_{p}}=\sum_{q=1}^{M} \sum_{q^{\prime}=1}^{M} \kappa_{q} \kappa_{q^{\prime}}\left(\mathbf{V}_{q}^{*} \mathbf{U}_{q^{\prime}}\right)\left(\mathbf{V}_{q^{\prime}}^{\mathbf{T}} \overline{\mathbf{U}_{p}}\right) \overline{\mathbf{U}_{q}}=\kappa_{p}^{2}\left|\mathbf{V}_{p}^{*} \mathbf{U}_{p}\right|^{2} \overline{\mathbf{U}_{p}}+R_{p},
$$

where we have set

$$
R_{p}=\sum_{q, q^{\prime} \in\{1, \ldots, M\},\left(q, q^{\prime}\right) \neq(p, p)} \kappa_{q} \kappa_{q^{\prime}}\left(\mathbf{V}_{q}^{*} \mathbf{U}_{q^{\prime}}\right)\left(\mathbf{V}_{q^{\prime}}^{\mathbf{T}} \overline{\mathbf{U}_{p}}\right) \overline{\mathbf{U}_{q}} .
$$

But Proposition 4.5 implies that $\lim _{N \rightarrow+\infty}\left|\mathbf{V}_{p}^{*} \mathbf{U}_{p}\right|^{2}=\left(\frac{\alpha}{2 \pi}\right)^{2}$ and that $\left\|R_{p}\right\|=o\left(\left\|U_{p}\right\|\right)$ (where the norms are taken in $\mathbb{C}^{N}$ ). Therefore, relation (4.34) shows that $\overline{\mathbf{U}_{p}}$ constitutes an approximate eigenvector of the time reversal matrix associated with the approximate eigenvalue $\lambda_{p}=\left(\frac{\alpha \kappa_{p}}{2 \pi}\right)^{2}$.

We are now in position to tackle the second goal of this subsection, namely the selective focusing properties of these approximate eigenvectors. If we note that the components of the eigenvector $\overline{\mathbf{U}_{p}}$ are

$$
\overline{U_{m}^{p}}=\overline{u_{m}^{I}\left(s_{p}\right)}=-\frac{1}{2 i \beta_{m}} \exp \left(-i \beta_{m} s_{p}^{1}\right) \phi_{m}\left(s_{p}^{2}\right), \quad \forall 1 \leq m \leq N,
$$

we see that according to formula (2.5), the incident field generated by each approximate eigenvector is

$$
u_{p}^{I}(x)=\sum_{m=1}^{N}-\frac{1}{2 i \beta_{m}} \exp \left[i \beta_{m}\left(x_{1}-s_{p}^{1}\right)\right] \phi_{m}\left(s_{p}^{2}\right) \phi_{m}\left(x_{2}\right) .
$$

The next Proposition provides a justification of the selective focusing properties of the D.O.R.T. method for large values of $N$ (the subscript $p$ refering to the scatterer under consideration has been dropped in this Proposition for the sake of clarity).

Proposition 4.7. Given $s=\left(s_{1}, s_{2}\right) \in(0, L) \times(0, d)$, let $u^{I}(x)$ be defined for all $x=\left(x_{1}, x_{2}\right) \in(0, L) \times(0, d)$ by

$$
u^{I}(x)=\sum_{m=1}^{N} \frac{1}{2 i \beta_{m}} \exp \left[i \beta_{m}\left(x_{1}-s_{1}\right)\right] \phi_{m}\left(s_{2}\right) \phi_{m}\left(x_{2}\right) .
$$

Then, given $\theta \in(0,1]$ and $\eta>0$, and under assumption (4.30), $u^{I}(x)$ satisfies the following properties :

i) Let $\alpha$ be given by Proposition 4.3. Then

$$
\lim _{N \rightarrow+\infty}\left|u^{I}(s)\right|=\alpha /(2 \pi)
$$


ii) As $N \rightarrow+\infty$, the estimate

$$
u^{I}\left(s_{1}, x_{2}\right)=\mathcal{O}\left(N^{-\theta / 2}\right),
$$

holds uniformly for $x_{2} \in(0, d)$ satisfying

$$
\left|\left(x_{2}-s_{2}\right) / d\right| \geq \eta N^{(\theta-1) / 2} .
$$

iii) As $N \rightarrow+\infty$, the estimate

$$
u^{I}(x)=\mathcal{O}\left(N^{-1 / 4}\right)+\mathcal{O}\left(N^{-\theta / 2}\right),
$$

holds uniformly for $x=\left(x_{1}, x_{2}\right) \in(0, L) \times(0, d)$ satisfying

$$
\left|\left(x_{1}-s_{1}\right) / d\right| \geq \eta N^{\theta-1}
$$

Proof. Let us set as in the proof of Proposition 4.5: $X_{1}=\pi\left(x_{1}-s_{1}\right) / d$, $X_{2}^{ \pm}=\pi\left(x_{2} \pm s_{2}\right) / d$ and $\beta_{m}^{N}=d \beta_{m} / \pi=\sqrt{(k d / \pi)^{2}-m^{2}}$. Relation (4.36) can be written

$$
\begin{aligned}
& u^{I}(x)=-\frac{1}{4 i \pi} \sum_{m=1}^{N} \frac{1}{\beta_{m}^{N}} \exp \left(i \beta_{m}^{N} X_{1}\right) {\left[\exp \left(i m X_{2}^{+}\right)+\exp \left(-i m X_{2}^{+}\right)\right.} \\
&\left.-\exp \left(i m X_{2}^{-}\right)-\exp \left(-i m X_{2}^{-}\right)\right] .
\end{aligned}
$$

Therefore, with the notation of Subsection 4.2 and setting $\tau_{N}=k d / \pi$, we have

$$
u^{I}(x)=-\frac{1}{4 i \pi}\left[\rho_{N}\left(X_{1}, X_{2}^{+}\right)+\rho_{N}\left(X_{1},-X_{2}^{+}\right)-\rho_{N}\left(X_{1}, X_{2}^{-}\right)-\rho_{N}\left(X_{1},-X_{2}^{-}\right)\right] .
$$

Assertions $i$ ), $i$ i) and $i$ ii) follow then from Proposition 4.3. More precisely, for assertion $i$ ), we have $x=s$ and thus $X_{1}=0, X_{2}^{-}=0$ and $X_{2}^{+}=2 \pi s_{2} / d$. Therefore

$$
\left|u^{I}(x)\right|=\frac{1}{4 \pi}\left|\rho_{N}\left(0, X_{2}^{+}\right)+\rho_{N}\left(0,-X_{2}^{+}\right)-2 \rho_{N}(0,0)\right|
$$

and the result follows from assertions $i$ ) and $i i$ ) of Proposition 4.3. In the case of assertion $i i)$, we have $X_{1}=0$, and $X_{2}^{ \pm}=\pi\left(x_{2} \pm s_{2}\right) / d$ and thus, assertion $\left.i i\right)$ of Proposition 4.3 implies that

$$
\rho_{N}\left(0, \pm X_{2}^{+}\right)=\mathcal{O}\left(N^{-1 / 2}\right), \quad \rho_{N}\left(0, \pm X_{2}^{-}\right)=\mathcal{O}\left(N^{-\theta / 2}\right),
$$

The above relations, together with (4.42), yield (4.38). Finally, (4.40) follows directly from the corresponding assertion iii) of Proposition 4.3.

Let us make a few comments concerning the consequences of Proposition 4.7 with regards to the selective focusing properties of the D.O.R.T. method. Assertions $i$ ) and ii) in Proposition 4.7 show that the expected focusing properties hold for large values of $N$ on the vertical line containing the source. Assertion iii) deals with the case in which $x_{1}-s_{1} \neq 0$. It shows that the acoustic field generated by the approximate eigenvector $\overline{\mathbf{U}_{p}}$ at a point $x$ converges to 0 as $N \rightarrow+\infty$ if the horizontal distance between $x$ and the scatterer $p$ is (at least) of order $d / N^{1-\theta}$. 


\section{Numerical results}

In this Section, we present some numerical experiments to illustrate the selective focusing properties investigated in Section 4. Our computations are realized using a finite element PDE Toolbox developed for SCILAB (see [16]). In order to compute the scattering matrix $\mathbb{S}$, we have to solve $N$ diffraction problems of the type (2.6), each one of them corresponding to the incident field $\Phi_{n}^{+}(x)=\exp \left(i \beta_{n} x_{1}\right) \phi_{n}\left(x_{2}\right)$, $1 \leq n \leq N$. Since problem (2.6) is set in the unbounded domain $\Omega=\Omega_{\infty} \backslash \overline{\mathcal{O}}$, we use for its numerical resolution an alternative -and equivalent- formulation of (2.6) set in the bounded domain $\Omega_{0}=\left\{\left(x_{1}, x_{2}\right) \in(\ell, L) \times(0, d), x \notin \overline{\mathcal{O}}\right\}$. To achieve this, we use on the vertical boundaries $\Gamma_{B}$ and $\Gamma_{C}$ (cf. Figure 1) transparent boundary conditions based on the Dirichlet-to-Neumann operators. A $\mathbb{P}^{1}$-piecewise finite element approximation of the problem satisfied by the total field leads then to solve the linear system

$$
\mathbb{A}_{h} X_{h}:=\left(\mathbb{K}_{h}-k^{2} \mathbb{M}_{h}-\mathbb{L}_{h}\right) X_{h}=B_{h},
$$

where $\mathbb{K}_{h}$ and $\mathbb{M}_{h}$ denote respectively the stiffness and the mass matrices and where $\mathbb{L}_{h}$ approximates the Dirichlet-to-Neumann transparent boundary conditions:

$$
\mathbb{L}_{h}^{I J}= \begin{cases}\sum_{m=1}^{N} i \beta_{m}\left(w_{I}, \phi_{m}\right)\left(w_{J}, \phi_{m}\right) & \text { if } M_{I}, M_{J} \in \Gamma_{C} \text { or } M_{I}, M_{J} \in \Gamma_{B} \\ 0 & \text { otherwise. }\end{cases}
$$

where $w_{I}$ denotes the hat function associated with the node $M_{I}$ and $\left(w_{I}, \phi_{m}\right)=$ $\int_{0}^{d} w_{I}\left(x_{2}\right) \phi_{m}\left(x_{2}\right) d x_{2}$.

Remark 5.1. Note that in formula (5.2), the infinite sum appearing in the continuous problem has been cut off to take into account only the propagating modes.

To compute the $N \times N$ scattering matrix $\mathbb{S}_{h}$, we note that its column $n$ $(n=1, \ldots, N)$ can be obtained by solving the linear system (5.1) for the particular right hand side $B_{h}^{I}=-2 i \beta_{n} \exp \left(i \beta_{n} \ell\right)\left(w_{I}, \phi_{n}\right)$ if $M_{I} \in \Gamma_{C}$ and $B_{h}^{I}=0$ otherwise. Finally, according to Remark $2.3, S_{m n}^{h}$ can be computed by the relation

$$
S_{m n}^{h}=\left(\left(u_{h}^{T}-u_{h}^{I}\right)_{\mid \Gamma_{A}}, \phi_{m}\right)=\exp \left(i \beta_{m} \ell\right)\left(\left(u_{h}^{T}-u_{h}^{I}\right)_{\mid \Gamma_{C}}, \phi_{m}\right) .
$$

The approximate discrete time reversal matrix $\mathbb{T}_{h}$ is then deduced from $\mathbb{S}_{h}$ by computing $\mathbb{T}_{h}=\overline{\mathbb{S}_{h}} \mathbb{S}_{h}$.

The case of one scatterer and three scatterers are successively considered. In the first situation, we mainly focus on the influence of the size of the scatterer on the eigenvalues of the scattering matrix. In the case of three scatterers, we investigate the focusing properties of the incident fields corresponding to the eigenvectors associated with the four largest eigenvalues. In particular, the effect of the number $N$ of propagating modes of the waveguide is studied. The waveguide considered for our numerical experiments is of height $d=10.2$. The vertical boundaries $\Gamma_{B}$ and $\Gamma_{C}$ on which the transparent conditions are written are located respectively at $x_{1}=L=13$ and $x_{1}=\ell=9$ (recall that the mirror is located at $x_{1}=0$; cf. Figure 1 ).

In the first experiment, we consider a spherical scatterer of radius $r$ centered at the point $s=(\ell+1.03(L-\ell) / 3 ; 0.99 d / 5)$. We assume that the wavelength is $\lambda=1$ (i.e. $k=2 \pi$ ). The mesh used contains about 240000 triangles and the mesh size is 
$h=0.025$. Under these assumptions, Table 1 shows the dependence of the ratio of the two largest eigenvalues of the scattering matrix with respect to the radius $r$ of the scatterer. As expected, there is only one significant eigenvalue when the scatterer is small enough.

Table 1. Ratio between the two largest eigenvalues of the scattering matrix as function of the size of the scatterer ( $d$ is the height of the waveguide).

\begin{tabular}{lllll}
\hline$r$ & $d / 50$ & $d / 100$ & $d / 150$ & $d / 200$ \\
\hline$\lambda_{1} / \lambda_{2}$ & 2.1 & 11.2 & 34.9 & 80.6 \\
\hline
\end{tabular}

In the second experiment, we assume that the waveguide contains three spherical scatterers of radii $r_{1}=d / 200, r_{2}=d / 400$ and $r_{3}=d / 800$ centered respectively at $s_{1}=(\ell+4(L-\ell) / 5 ; 9 d / 10), s_{2}=(\ell+4(L-\ell) / 5 ; d / 10)$ and $s_{3}=(\ell+(L-\ell) / 2 ; d / 2)$. Figure 2 represents the incident fields corresponding to the eigenvectors associated with the four largest eigenvalues of the scattering matrix in the case of a wavelength $\lambda=2$ (i.e. $k=\pi$ ). The number of propagating modes $N$ in this case is equal to 10 . Selective focusing is observed as predicted by the theory: the eigenvector associated with the largest eigenvalue focuses on the biggest scatterer and so on. If the wavelength is reduced to $\lambda=0.5$, i.e. if $N$ is increased to 40, we can see in Figure 3 that the quality of the focusing is clearly improved.
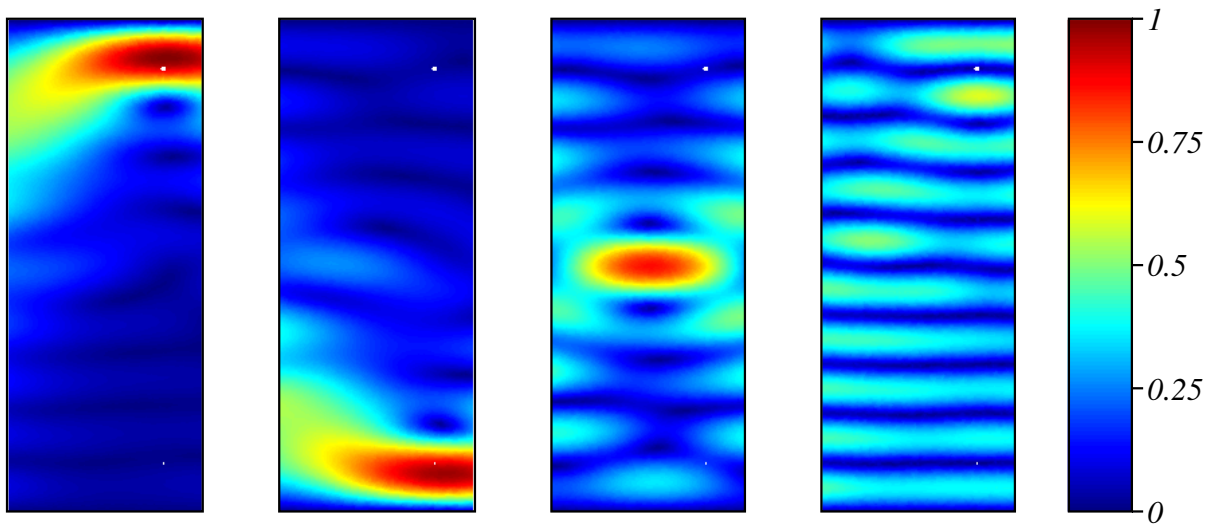

Figure 2. Incident fields corresponding to eigenvectors associated with the four largest eigenvalues of the scattering matrix for $N=10$ propagating modes.

\section{Appendix. Scattering by small obstacles in a two-dimensional acoustic waveguide}

This appendix is devoted to the proof of Proposition 6.1 stated below, which constitutes the main ingredient of the proof of Proposition 4.1. Indeed, it provides the asymptotics for the scattering problem by small obstacles in a two-dimensional waveguide. Let us emphasize that scattering by small inhomogeneities has been 

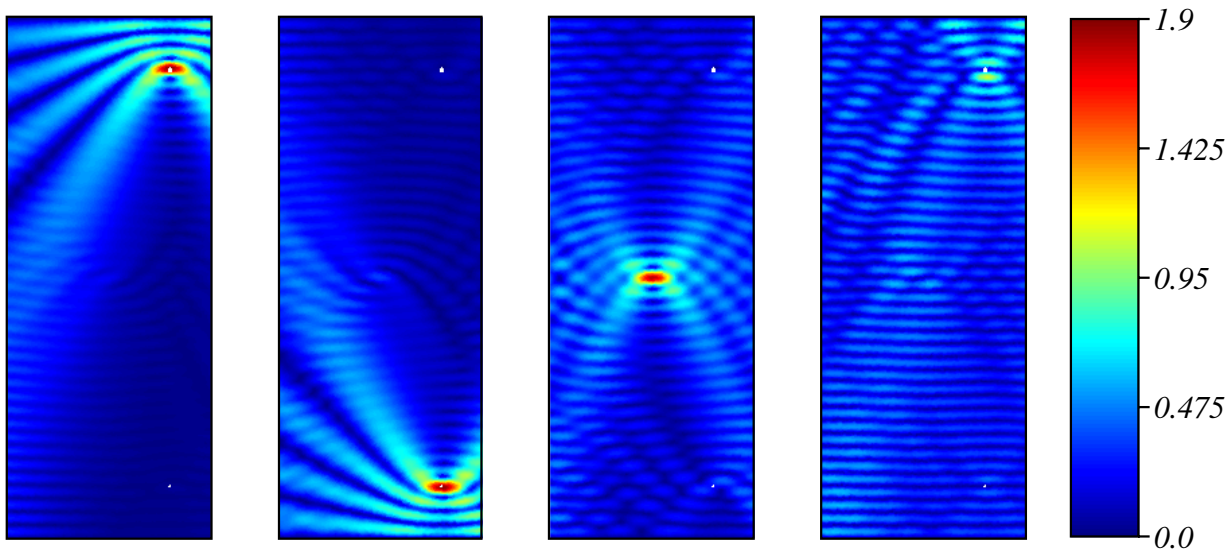

Figure 3. Incident fields corresponding to eigenvectors associated with the four largest eigenvalues of the scattering matrix for $N=40$ propagating modes.

thoroughly investigated in the last few years (see [4] for a detailed presentation and more references), in particular to develop efficient algorithms for recovering the localization and the shape of the inhomogeneities (cf. [2], [3]).

A similar result to Proposition 6.1 is proved in the Appendix of [13] in the case of scattering by small obstacles in $\mathbb{R}^{3}$. Nevertheless, since the geometry considered here is two-dimensional, one can not adapt the proof given there. Indeed, the singularities of the Green's functions are different in the two cases, leading to different non vanishing terms in the limiting process. An additional and crucial difference comes from a technical difficulty that appears in the $2 \mathrm{D}$ case. Indeed, contrarily to the $3 \mathrm{D}$ case, the free space Green's function of $\Delta+k^{2}$ in $2 \mathrm{D}$, namely $\frac{i}{4} H_{0}^{1}(k|x-y|)$, does not converge as $k \rightarrow 0$ to the free space Green's function of the Laplace operator $\frac{1}{2 \pi} \ln \frac{1}{|x-y|}$, due to the presence of a term involving $\ln k$. This property makes the low frequency analysis of scattering problems in $2 \mathrm{D}$ more intricate than in $3 \mathrm{D}$. In order to overcome this difficulty, which also occurs in the context of scattering by small scatterers, we use an approach that was first used in [19] and then extended in [14] for the analysis of low frequency asymptotics. In this approach, we seek the solution of the scattering problem in the form of an ansatz (see (6.2)) which is a suitable linear combination of single and double layer potentials. The key point in choosing this linear combination is to obtain a cancellation of the undesired terms (namely those involving $\ln e$ ) in the limiting process e $\rightarrow 0$. Let us also emphasize that this limiting process is performed on an Fredholm type integral equation of the second kind.

In the problem investigated here, there are two main difficulties. As mentioned above, the first one is related to the fact that we are dealing with a $2 \mathrm{D}$ problem. The second one is that we consider several scatterers and thus, we have to see if their interactions need to be taken into account in the limit model. For the sake of clarity, we separated these two difficulties in the rest of the Appendix by considering first the case of a single scatterer before studying the case of multiple scatterers. 
The notation used are those of the beginning of Section 4.

Proposition 6.1. Consider a family of obstacles $\left\{\mathcal{O}_{p}^{\varepsilon} ; 1 \leq p \leq M\right\}$, where each scatterer $\mathcal{O}_{p}^{\varepsilon}$ is obtained from a reference open set $\mathcal{O}_{p}$ (which is assumed to contain the origin) by applying a dilation of ratio $\varepsilon$ centered at a given point $s_{p} \in \mathbb{R}^{2}$ :

$$
\mathcal{O}_{p}^{\varepsilon}=\left\{x \in \mathbb{R}^{2} ; \xi=\left(x-s_{p}\right) / \varepsilon \in \mathcal{O}_{p}\right\} .
$$

Let $\Gamma_{p}^{\varepsilon}$ (respectively $\left.\Gamma_{p}\right)$ denote the boundary of $\mathcal{O}_{p}^{\varepsilon}$ (respectively $\left.\mathcal{O}_{p}\right)$, and set

$$
\Gamma^{\varepsilon}=\bigcup_{1 \leq p \leq M} \Gamma_{p}^{\varepsilon} \quad \mathcal{O}^{\varepsilon}=\bigcup_{1 \leq p \leq M} \mathcal{O}_{p}^{\varepsilon} \quad \Gamma=\bigcup_{1 \leq p \leq M} \Gamma_{p} \quad \mathcal{O}=\bigcup_{1 \leq p \leq M} \mathcal{O}_{p} .
$$

Denote by $u_{\varepsilon}^{D}$ be the solution of the following exterior Dirichlet problem

$$
\left\{\begin{array}{l}
\Delta u_{\varepsilon}^{D}+k^{2} u_{\varepsilon}^{D}=0 \text { in } \Omega_{\infty} \backslash \overline{\mathcal{O}^{\varepsilon}} \\
u_{\varepsilon}^{D}=-u^{I} \text { on } \Gamma^{\varepsilon} \\
u_{\varepsilon}^{D}=0 \text { on } \Sigma_{\infty} \\
u_{\varepsilon}^{D} \text { outgoing. }
\end{array}\right.
$$

Around each reference obstacle $\mathcal{O}_{p}$, delimit a bounded part $D_{p}$ of its exterior by a fictitious boundary $\Sigma_{p}$ which does not intersect $\Gamma_{p}$. We denote by $D_{p}^{\varepsilon}$ and $\Sigma_{p}^{\varepsilon}$ the images of $D_{p}$ and $\Sigma_{p}$ by the dilation $x=\varepsilon \xi+s_{p}$ and we set

$$
D^{\varepsilon}=\bigcup_{1 \leq p \leq M} D_{p}^{\varepsilon} \quad \Sigma^{\varepsilon}=\bigcup_{1 \leq p \leq M} \Sigma_{p}^{\varepsilon} \quad D=\bigcup_{1 \leq p \leq M} D_{p} \quad \Sigma=\bigcup_{1 \leq p \leq M} \Sigma_{p}
$$

For all $x=s_{p}+\varepsilon \xi \in D_{p}^{\varepsilon}$, with $\xi \in D_{p}$, we set

$$
\varphi_{p}^{\varepsilon}(\xi)=u_{\varepsilon}^{D}(x)
$$

Finally, let

$$
\varphi_{p}^{0}=-u^{I}\left(s_{p}\right) \Theta_{p}
$$

where $\Theta_{p}$ is the static potential solution to

$$
\left\{\begin{array}{l}
\Delta \Theta_{p}=0 \text { in } \mathbb{R}^{2} \backslash \mathcal{O}_{p} \\
\Theta_{p}=1 \text { on } \Gamma_{p} \\
\Theta_{p}=O(1) \text { for }|x| \rightarrow \infty
\end{array}\right.
$$

Then, the following estimate holds true

$$
\left\|\varphi_{p}^{\varepsilon}-\varphi_{p}^{0}\right\|_{H^{1}\left(D_{p}\right)}=O\left(\frac{1}{\ln \mathrm{e}}\right), \quad \forall 1 \leq p \leq M .
$$

\subsection{Single scatterer}

We first prove Proposition 6.1 in the case of a single scatterer. Therefore, we drop in this Subsection the subscript $p$ referring to the number of the scatterer. The idea of the proof is to rewrite the scattering problem (6.1) as an integral equation on $\Gamma$. We seek the solution of $u_{\varepsilon}^{D}$ of (6.1) in the form

$$
\begin{aligned}
u_{\varepsilon}^{D}(x)=\int_{\Gamma^{\varepsilon}}\left[\mathrm{e} \frac{\partial G}{\partial \nu_{y}}(x, y)+\right. & \left.\left(1-\frac{2 \pi}{\ln \mathrm{e}}\right) G(x, y)\right] \Psi^{\mathrm{e}}(y) d \gamma_{y} \\
& -\frac{1}{\left|\Gamma^{\mathrm{e}}\right|}\left(\int_{\Gamma^{\varepsilon}} G(x, y) d \gamma_{y}\right)\left(\int_{\Gamma^{\varepsilon}} \Psi^{\mathrm{e}}(y) d \gamma_{y}\right) \quad x \in D^{\mathrm{e}},
\end{aligned}
$$


for some unknown density $\Psi^{\mathrm{e}} \in L^{2}\left(\Gamma^{\mathrm{e}}\right)$.

Clearly, the above potential is an outgoing solution of the Helmholtz equation in $\Omega_{\infty} \backslash \overline{\mathcal{O}^{\varepsilon}}$ that vanishes on $\partial \Omega_{\infty}$. By the jump relations for single and double layer potentials (see [9]), the boundary condition in (6.1) is satisfied if and only if

$$
\begin{aligned}
\frac{\mathrm{e}}{2} \Psi^{\mathrm{e}}(x)+ & \int_{\Gamma^{\varepsilon}}\left[\mathrm{e} \frac{\partial G}{\partial \nu_{y}}(x, y)+\left(1-\frac{2 \pi}{\ln \mathrm{e}}\right) G(x, y)\right] \Psi^{\mathrm{e}}(y) d \gamma_{y} \\
& -\frac{1}{\left|\Gamma^{\mathrm{e}}\right|}\left(\int_{\Gamma^{\varepsilon}} G(x, y) d \gamma_{y}\right)\left(\int_{\Gamma^{\varepsilon}} \Psi^{\mathrm{e}}(y) d \gamma_{y}\right)=-u^{I}(x), \quad x \in \Gamma^{\mathrm{e}}
\end{aligned}
$$

In order to work in a functional framework independent of $\varepsilon$, we set for all $x, y \in D^{\mathrm{e}}$ :

$$
\left\{\begin{array}{l}
\xi=(x-s) / \varepsilon \\
\eta=(y-s) / \varepsilon
\end{array}\right.
$$

and we define

$$
\left\{\begin{array}{lll}
\varphi^{\varepsilon}(\xi)=u_{\varepsilon}^{D}(x) & \xi \in D, & x \in D^{\mathrm{e}} \\
G^{\mathrm{e}}(\xi, \eta)=G(x, y), & \xi, \eta \in D, & x, y \in D^{\mathrm{e}} \\
\psi^{\varepsilon}(\xi)=\mathrm{e}^{\varepsilon}(x), & \xi \in \Gamma, & x \in \Gamma^{\mathrm{e}} \\
f^{\mathrm{e}}(\xi)=-2 u_{I}(x), & \xi \in \Gamma, & x \in \Gamma^{\mathrm{e}}
\end{array}\right.
$$

Noting that for all $(\xi, \eta) \in D \times D$ and $(x, y) \in D^{\mathrm{e}} \times D^{\mathrm{e}}$ related by (6.4) we have

$$
\nabla_{\eta} G^{\mathrm{e}}(\xi, \eta)=\mathrm{e} \nabla_{y} G(x, y)
$$

a straightforward calculation shows that the ansatz (6.2) can be written in the new variables $(\xi, \eta)$ as

$$
\varphi^{\varepsilon}=\mathcal{L}^{\mathrm{e}}\left(\psi^{\mathrm{e}}\right)
$$

where we have set for $\mathrm{e}>0$

$$
\begin{aligned}
\mathcal{L}^{\mathrm{e}} \psi(\xi)=\int_{\Gamma}\left[\frac{\partial G^{\mathrm{e}}}{\partial \nu_{\eta}}(\xi, \eta)\right. & \left.+\left(1-\frac{2 \pi}{\ln \mathrm{e}}\right) G^{\mathrm{e}}(\xi, \eta)\right] \psi(\eta) d \gamma_{\eta} \\
& -\frac{1}{|\Gamma|}\left(\int_{\Gamma} G^{\mathrm{e}}(\xi, \eta) d \gamma_{\eta}\right)\left(\int_{\Gamma} \psi(\eta) d \gamma_{\eta}\right), \quad \xi \in D
\end{aligned}
$$

Moreover, the integral equation (6.3) written in the new variables $(\xi, \eta)$ reads

$$
\left(I+L^{\mathrm{e}}\right) \psi^{\mathrm{e}}=f^{\mathrm{e}},
$$

where

$$
\begin{aligned}
L^{\mathrm{e}} \psi(\xi)=2 \int_{\Gamma}\left[\frac{\partial G^{\mathrm{e}}}{\partial \nu_{\eta}}\right. & \left.(\xi, \eta)+\left(1-\frac{2 \pi}{\ln \mathrm{e}}\right) G^{\mathrm{e}}(\xi, \eta)\right] \psi(\eta) d \gamma_{\eta} \\
& -\frac{2}{|\Gamma|}\left(\int_{\Gamma} G^{\mathrm{e}}(\xi, \eta) d \gamma_{\eta}\right)\left(\int_{\Gamma} \psi(\eta) d \gamma_{\eta}\right), \quad \xi \in \Gamma .
\end{aligned}
$$

It is well known that $\mathcal{L}^{\mathrm{e}} \in \mathcal{L}\left(L^{2}(\Gamma), H^{1}(D)\right)$ and that $L^{\mathrm{e}} \in \mathcal{L}\left(L^{2}(\Gamma)\right)$ for all e $>0$. 
Let now $g_{0}(x, y)=-(2 \pi)^{-1} \ln |x-y|$ denote the Green function of the Laplace operator in $\mathbb{R}^{2}$. We introduce then the operators $\mathcal{L}^{0} \in \mathcal{L}\left(L^{2}(\Gamma), H^{1}(D)\right)$ and $L^{0} \in \mathcal{L}\left(L^{2}(\Gamma)\right)$ by setting for all $\psi \in L^{2}(\Gamma)$ :

$$
\begin{aligned}
\mathcal{L}^{0} \psi(\xi)=\int_{\Gamma}\left[\frac{\partial g_{0}}{\partial \nu_{\eta}}(\xi, \eta)+g_{0}(\xi, \eta)+1\right] \psi(\eta) d \gamma_{\eta} & \\
& -\frac{1}{|\Gamma|}\left(\int_{\Gamma} g_{0}(\xi, \eta) d \gamma_{\eta}\right)\left(\int_{\Gamma} \psi(\eta) d \gamma_{\eta}\right), \quad \xi \in D . \\
L^{0} \psi(\xi)=2 \int_{\Gamma}\left[\frac{\partial g_{0}}{\partial \nu_{\eta}}(\xi, \eta)\right. & \left.+g_{0}(\xi, \eta)+1\right] \psi(\eta) d \gamma_{\eta} \\
& -\frac{2}{|\Gamma|}\left(\int_{\Gamma} g_{0}(\xi, \eta) d \gamma_{\eta}\right)\left(\int_{\Gamma} \psi(\eta) d \gamma_{\eta}\right), \quad \xi \in \Gamma
\end{aligned}
$$

Then, we have the following result.

Lemma 6.2. With the notation above, we have for e small enough:

$$
\begin{gathered}
\left\|L^{\mathrm{e}}-L^{0}\right\|_{\mathcal{L}\left(L^{2}(\Gamma)\right)}=\mathcal{O}\left(\frac{1}{\ln \mathrm{e}}\right) \\
\left\|\left(I+L^{\mathrm{e}}\right)^{-1}-\left(I+L^{0}\right)^{-1}\right\|_{\mathcal{L}\left(L^{2}(\Gamma)\right)}=\mathcal{O}\left(\frac{1}{\ln \mathrm{e}}\right),
\end{gathered}
$$

and

$$
\left\|\mathcal{L}^{\mathrm{e}}-\mathcal{L}^{0}\right\|_{\mathcal{L}\left(L^{2}(\Gamma), H^{1}(D)\right)}=\mathcal{O}\left(\frac{1}{\ln \mathrm{e}}\right) .
$$

Proof. The Green function of the waveguide admits the decomposition $G=g+R$, where $g(x, y)=(i / 4) H_{0}^{(1)}(k|x-y|)$ denotes the Green's function of $\Delta+k^{2}$ in $\mathbb{R}^{2}$ and where the function $R$ has continuous derivatives of arbitrary order on $\Omega_{\infty} \times \Omega_{\infty}$. Therefore, using the asymptotics of the Hankel function $H_{0}^{(1)}$ and its gradient for small arguments (see [1]), one obtains that

$$
\left\{\begin{array}{l}
G^{\mathrm{e}}(\xi, \eta)=g_{0}(\xi, \eta)-\frac{1}{2 \pi} \ln \mathrm{e}+\kappa+\mathcal{O}(\mathrm{e}) \\
\nabla_{\eta} G^{\mathrm{e}}(\xi, \eta)=\nabla_{\eta} g_{0}(\xi, \eta)+\mathcal{O}(\mathrm{e})
\end{array}\right.
$$

where $\kappa=H(s, s)+\frac{i}{4}-\frac{c+\ln (k / 2)}{2 \pi}$, where $c=0.5772 \ldots$ denotes Euler's constant. Formulas (6.13) hold uniformly in any compact subset of $\bar{D} \times \bar{D}$ which does not contain points of the diagonal. Relation (6.10) follows then from the substitution of the asympotics (6.13) into the expression (6.9) of $L^{\mathrm{e}}$. The estimate (6.11) can be derived from (6.10) by using a Neumann series argument. Finally, one can easily check that the asymptotics (6.13) imply the existence of a constant $C>0$ such that for all $\psi \in L^{2}(\Gamma)$

$$
\left\{\begin{array}{l}
\left\|\mathcal{L}^{\mathrm{e}} \psi-\mathcal{L}^{0} \psi\right\|_{L^{2}(D)} \leq \frac{C}{\ln \mathrm{e}}\|\psi\|_{L^{2}(\Gamma)} \\
\left\|\nabla\left(\mathcal{L}^{\mathrm{e}} \psi-\mathcal{L}^{0} \psi\right)\right\|_{L^{2}(D)} \leq C \mathrm{e}\|\psi\|_{L^{2}(\Gamma)}
\end{array}\right.
$$

and therefore (6.12) holds true. 
We are now in position to prove Proposition 6.1 in the case of one scatterer. Let $\psi^{0}$ be the solution of the integral equation

$$
\left(I+L^{0}\right) \psi^{0}=f^{0} .
$$

where $f^{0} \in L^{2}(\Gamma)$ is the constant fonction taking the value $-2 u^{I}(s)$. Noting that

$$
\psi^{\mathrm{e}}-\psi^{0}=\left(I+L^{\mathrm{e}}\right)^{-1} f^{\mathrm{e}}-\left(I+L^{0}\right)^{-1} f^{0},
$$

and using the estimate (6.11) together with the fact that the function $f^{\mathrm{e}}$ defined by (6.5) satisfies $\left\|f^{\mathrm{e}}-f^{0}\right\|_{L^{2}(\Gamma)}=\mathcal{O}(\mathrm{e})$, we obtain that for e small enough, there holds

$$
\left\|\psi^{\mathrm{e}}-\psi^{0}\right\|_{L^{2}(\Gamma)}=\mathcal{O}\left(\frac{1}{\ln \mathrm{e}}\right) .
$$

Let $\varphi^{\mathrm{e}}$ be the potential given by (6.2) and set $\varphi^{0}=-u^{I}(s) \Theta$, where $\Theta$ is the static potential defined by

$$
\left\{\begin{array}{l}
\Delta \Theta=0 \text { in } \mathbb{R}^{2} \backslash \mathcal{O}, \\
\Theta=1 \text { on } \Gamma, \\
\Theta=O(1) \text { for }|x| \rightarrow \infty .
\end{array}\right.
$$

As it is checked in [14], we have

$$
\varphi^{0}=\mathcal{L}^{0} \psi^{0}
$$

and consequently

$$
\varphi^{\mathrm{e}}-\varphi^{0}=\mathcal{L}^{\mathrm{e}} \psi^{\mathrm{e}}-\mathcal{L}^{0} \psi^{0}=\left(\mathcal{L}^{\mathrm{e}}-\mathcal{L}^{0}\right) \psi^{\mathrm{e}}+\mathcal{L}^{0}\left(\psi^{\mathrm{e}}-\psi^{0}\right),
$$

Thus, by using relations (6.12) and (6.15), we get that

$$
\left\|\varphi^{\mathrm{e}}-\varphi^{0}\right\|_{H^{1}(D)}=\mathcal{O}\left(\frac{1}{\ln \mathrm{e}}\right)
$$

for e small enough, and the proof is complete.

\subsection{Multiple scatterers}

We come back to the case of multiple scaterrers and we use the notations of Proposition 6.1 , in which the subscript $p=1, \ldots, M$ refers to the number of the scatterer. Using the same ansatz as the one used in Subsection 6.1, solving the scattering problem (6.1) amounts now to solve a family of $M$ coupled integral equations written on the boundaries $\Gamma_{p}, p=1, \ldots, M$ having for unknows the densities

$$
\psi^{\mathrm{e}}:=\left(\psi_{1}^{\mathrm{e}}, \ldots, \psi_{M}^{\mathrm{e}}\right) \in \mathcal{H}:=L^{2}\left(\Gamma_{1}\right) \times \cdots \times L^{2}\left(\Gamma_{M}\right) .
$$

More precisely, these integral equations can still be written in the reduced variables $\left(\xi_{p}, \eta_{p}\right)$ in the abstract form (6.8), namely

$$
\left(I+L^{\mathrm{e}}\right) \psi^{\mathrm{e}}=f^{\mathrm{e}},
$$

provided we use the following notations: 
- the operator $L^{\mathrm{e}} \in \mathcal{L}(\mathcal{H}, \mathcal{H})$ is defined through its block decomposition

$$
L^{\mathrm{e}} \psi=\left(\begin{array}{ccc}
L_{11}^{\mathrm{e}} & \ldots & L_{1 M}^{\mathrm{e}} \\
\vdots & & \vdots \\
L_{M 1}^{\mathrm{e}} & \ldots & L_{M M}^{\mathrm{e}}
\end{array}\right),
$$

in which $L_{p q}^{\mathrm{e}} \in \mathcal{L}\left(L^{2}\left(\Gamma_{q}\right), L^{2}\left(\Gamma_{p}\right)\right)$ is the integral operator defined for all density $\psi \in L^{2}\left(\Gamma_{q}\right)$ by

$$
\begin{gathered}
L_{p q}^{\mathrm{e}} \psi\left(\xi_{p}\right)=2 \int_{\Gamma_{q}}\left[\frac{\partial G_{p q}^{\mathrm{e}}}{\partial \nu_{\eta_{q}}}\left(\xi_{p}, \eta_{q}\right)+\left(1-\frac{2 \pi}{\ln \mathrm{e}}\right) G_{p q}^{\mathrm{e}}\left(\xi_{p}, \eta_{q}\right)\right] \psi\left(\eta_{q}\right) d \gamma_{\eta_{q}} \\
-\frac{2}{\left|\Gamma_{q}\right|}\left(\int_{\Gamma_{q}} G_{p q}^{\mathrm{e}}\left(\xi_{p}, \eta_{q}\right) d \gamma_{\eta}\right)\left(\int_{\Gamma_{q}} \psi\left(\eta_{q}\right) d \gamma_{\eta_{q}}\right), \quad \xi_{p} \in \Gamma_{p} .
\end{gathered}
$$

where

$$
G_{p q}^{\mathrm{e}}\left(\xi_{p}, \eta_{q}\right)=G\left(s_{p}+\mathrm{e} \xi_{p}, s_{q}+\mathrm{e} \eta_{q}\right), \quad \xi_{p}, \eta_{q} \in D_{p} \times D_{q} .
$$

- The data $f^{\mathrm{e}}$ are now given by $f^{\mathrm{e}}=\left(f_{1}^{\mathrm{e}}, \ldots, f_{M}^{\mathrm{e}}\right) \in \mathcal{H}$, with

$$
f_{p}^{\mathrm{e}}\left(\xi_{p}\right)=-2 u_{I}\left(s_{p}+\mathrm{e} \xi_{p}\right), \quad \xi_{p} \in \Gamma_{p} .
$$

The key point to extend the result of the previous Subsection to the case of many scatterers is that for $p \neq q$, the function $G_{p q}^{\mathrm{e}}$ admits the asymptotics

$$
\left\{\begin{array}{l}
G_{p q}^{\mathrm{e}}(\xi, \eta)=G\left(s_{p}, s_{q}\right)+\mathcal{O}(\mathrm{e}) \\
\nabla_{\eta} G_{p q}^{\mathrm{e}}(\xi, \eta)=\mathcal{O}(\mathrm{e})
\end{array}\right.
$$

Using the above relations, one can easily check that

$$
\left\|L_{p q}^{\mathrm{e}}\right\|_{\mathcal{L}\left(L^{2}\left(\Gamma_{q}\right), L^{2}\left(\Gamma_{p}\right)\right)}=\mathcal{O}\left(\frac{1}{\ln \mathrm{e}}\right), \quad \forall p \neq q .
$$

This relation implies that the (formal) limit of the family of integral equations (6.17) is given by $M$ decoupled integral equations, each one of them being set on $\Gamma_{p}$ :

$$
\left(I+L_{p}^{0}\right) \psi_{p}^{0}=f_{p}^{0},
$$

where

$$
\begin{aligned}
L_{p}^{0} \psi\left(\xi_{p}\right)=2 \int_{\Gamma_{p}} & {\left[\frac{\partial g_{0}}{\partial \nu_{\eta_{p}}}\left(\xi_{p}, \eta_{p}\right)+g_{0}\left(\xi_{p}, \eta_{p}\right)+1\right] \psi\left(\eta_{p}\right) d \gamma_{\eta_{p}} } \\
& -\frac{2}{\left|\Gamma_{p}\right|}\left(\int_{\Gamma} g_{0}\left(\xi_{p}, \eta_{p}\right) d \gamma_{\eta_{p}}\right)\left(\int_{\Gamma_{p}} \psi\left(\eta_{p}\right) d \gamma_{\eta_{p}}\right), \quad \xi_{p} \in \Gamma_{p} .
\end{aligned}
$$

and $f_{p}^{0} \in L^{2}\left(\Gamma_{p}\right)$ is the constant fonction taking the value $-2 u^{I}\left(s_{p}\right)$. The rest of the proof goes then exactly along the lines of the proof detailed in Subsection 6.1, by first establishing that $\left\|\psi^{\mathrm{e}}-\psi^{0}\right\|_{\mathcal{H}} \longrightarrow 0$ and then that

$$
\left\|\varphi_{p}^{\mathrm{e}}-\varphi_{p}^{0}\right\|_{H^{1}\left(D_{p}\right)} \longrightarrow 0, \quad p=1, \ldots, M
$$

with $\varphi_{p}^{0}=\mathcal{L}_{p}^{0} \psi_{p}^{0}=-u^{I}\left(s_{p}\right) \Theta_{p}$. 


\section{Acknowledgments}

The authors would like to thank Professor Patrick Sargos for helpful discussions on sums of exponentials and the referees for their valuable remarks, comments and suggestions.

\section{References}

[1] M. Abramowitz And I. A. Stegun, Handbook of mathematical functions with formulas, graphs, and mathematical tables, vol. 55 of National Bureau of Standards Applied Mathematics Series, U.S. Government Printing Office, Washington, D.C., 1964.

[2] H. Ammari, E. Iakovleva, And H. Kang, Reconstruction of a small inclusion in a twodimensional open waveguide, SIAM J. Appl. Math., 65 (2005), pp. 2107-2127 (electronic).

[3] H. Ammari, E. IAKovleva, And D. Lesselier, Two numerical methods for recovering small inclusions from the scattering amplitude at a fixed frequency, SIAM J. Sci. Comput., 27 (2005), pp. 130-158 (electronic).

[4] H. AMmARI AND H. KANG, Reconstruction of small inhomogeneities from boundary measurements, vol. 1846 of Lecture Notes in Mathematics, Springer-Verlag, Berlin, 2004.

[5] C. Bardos And M. Fink, Mathematical foundations of the time reversal mirror, Asymptot. Anal., 29 (2002), pp. 157-182.

[6] P. Blomgren, G. Papanicolaou, and H. Zhao, Super-resolution in time-reversal acoustics, J. Acoust. Soc. Am., 11 (2002), pp. 230-248.

[7] M. Cheney, D. IsAacson, and M. Lassas, Optimal acoustic measurements, SIAM J. Appl. Math., 61 (2001), pp. 1628-1647.

[8] R. E. Collin, Field theory of guided waves, 2nd Edition, IEEE Press Series on Electromagnetic Wave Theory, Inc., New York, 1991.

[9] D. L. Colton And R. Kress, Integral equation methods in scattering theory, Pure and Applied Mathematics (New York), John Wiley \& Sons Inc., New York, 1983. , A Wiley-Interscience Publication.

[10] D. V. Evans, M. Levitin, And D. Vassiliev, Existence theorems for trapped modes, J. Fluid Mech., 261 (1994), pp. 21-31.

[11] M. Fink, Time-reversal in acoustics, Contemp. Phys., 37 (1996), pp. 95-109.

[12] M. Fink AND C. PRADA, Eigenmodes of the time-reversal operator: A solution to selective focusing in multiple-target media, Wave Motion, 20 (1994), pp. 151-163.

[13] C. Hazard and K. Ramdani, Selective acoustic focusing using time-harmonic reversal mirrors, SIAM J. Appl. Math., 64 (2004), pp. 1057-1076.

[14] R. KRess, On the low wave number asymptotics for the two-dimensional exterior Dirichlet problem for the reduced wave equation, Math. Methods Appl. Sci., 9 (1987), pp. 335-341.

[15] T. Mast, A. Nachman, And R. WAAG, Focusing and imaging using the eigenfunctions of the scattering operator, J. Acoust. Soc. Am., 102 (1997), pp. 715-725.

[16] B. Pinçon and K. Ramdani, Scilab tools for pdes's : Application to time-reversal, in Proceedings CCA/ISIC/CACSD'04, IEEE, 2004.

[17] A. Ramm and G. Makrakis, Scattering by obstacles in acoustic waveguides, in Spectral and scattering theory (Newark, DE, 1997), Plenum, New York, 1998, pp. 89-109.

[18] G. Tenenbaum, Introduction to analytic and probabilistic number theory, vol. 46 of Cambridge Studies in Advanced Mathematics, Cambridge University Press, Cambridge, 1995.

[19] P. Werner, Low frequency asymptotics for the reduced wave equation in two-dimensional exterior spaces, Math. Methods Appl. Sci., 8 (1986), pp. 134-156. 